\title{
Quantum corrections to the spin-independent cross section of the inert doublet dark matter
}

\author{
Tomohiro Abe and Ryosuke Sato \\ Theory Center, Institute of Particle and Nuclear Studies, \\ High Energy Accelerator Research Organization (KEK), \\ Tsukuba, 305-0801, Japan \\ E-mail: abetomo@post.kek.jp, rsato@post.kek.jp
}

ABSTRACT: The inert Higgs doublet model contains a stable neutral boson as a candidate of dark matter. We calculate cross section for spin-independent scattering of the dark matter on nucleon. We take into account electroweak and scalar quartic interactions, and evaluate effects of scattering with quarks at one-loop level and with gluon at two-loop level. These contributions give an important effect for the dark matter mass to be around $m_{h} / 2$, because a coupling with the standard model Higgs boson which gives the leading order contribution should be suppressed to reproduce the correct amount of the thermal relic abundance in this mass region. In particular, we show that the dark matter self coupling changes the value of the spin-independent cross section significantly.

KeYwords: Beyond Standard Model, Cosmology of Theories beyond the SM

ArXiv EPrint: 1501.04161 


\section{Contents}

1 Introduction 1

2 Model 3

3 Spin-independent cross section 5

$\begin{array}{ll}3.1 & \text { At the leading order }\end{array}$

$\begin{array}{ll}3.2 & \text { At the next leading order }\end{array}$

4 Results 10

5 Conclusion and discussion $\quad 13$

$\begin{array}{ll}\text { A Shorthand notations } & 14\end{array}$

B One-loop box type diagrams $\quad 14$

B.1 Z boson contribution $\quad 15$

B.2 $W$ boson contribution $\quad 16$

$\begin{array}{lll}\text { C One-loop higgs vertex corrections } & 17\end{array}$

$\begin{array}{ll}\text { C.1 } Z \text { boson contribution } & 17\end{array}$

$\begin{array}{lll}\text { C.2 } W \text { boson contribution } & 18\end{array}$

D Gluon contribution at two-loop level $\quad 19$

$\begin{array}{ll}\text { D.1 Two-point functions in the gluon background field } & 19\end{array}$

D.2 Effective interaction for dark matter-gluon scattering 23

E Loop functions for radiative corrections $\quad \mathbf{2 5}$

E.1 One-loop vertex 25

E.2 One-loop box diagrams 26

E.3 Loop functions for dark matter-gluon scattering 26

$\begin{array}{ll}\text { E.3.1 Definitions of } X, Y \text { functions } & 26\end{array}$

E.3.2 $X, Y$ in $B, C, D$-function $\quad 27$

E.3.3 $C, D$ in $B_{0}$ and $\partial B_{0} / \partial q^{2} \quad 27$

\section{Introduction}

The discovery of the Higgs boson at the Large hadron collider (LHC) in 2012 [1, 2] is one of the biggest achievements of the standard model (SM). In spite of its success, the SM does not include a candidate of the dark matter which has many evidences for existing in our universe [3]. Hence, we need some extension of the SM to explain the dark matter as an elementary particle. 
The inert two-Higgs doublet model $[4,5]$ is a simple extension of the SM with a dark matter candidate. It was originally discussed in an analysis of electroweak symmetry breaking in the two Higgs doublet model by Deshpande and Ma [4], and recently, it draws attention as a model of dark matter [5]. In this model, an additional $\mathrm{SU}(2)_{L}$ doublet scalar field with $Y=1 / 2$, which is called inert doublet, and a $Z_{2}$ parity are introduced. Under this parity, all of the SM fields are even and the inert doublet is odd. Then the lightest neutral boson with the $Z_{2}$ odd charge becomes the dark matter candidate. The $Z_{2}$ odd particles have electroweak interaction and scalar quartic interactions with the SM Higgs boson. Thus, they are thermalized in the early universe, and the amount of the dark matter in the present universe is generated as a thermal relic [6-8].

The Higgs sector in the inert doublet model sometimes appears in a part of beyond the standard models, e.g., left-right Twin Higgs model [9-11], a composite Higgs model [12], a radiative seesaw model [13-15] and models of neutrino flavor with non-Abelian discrete symmetry [16-19]. Also, the inert doublet model is analyzed in contexts of strong first order electroweak phase transition [20-24], Coleman-Weinberg mechanism driven by the inert doublet [25], and inflation [26]. In spite of its simplicity, the inert doublet model has rich phenomenology. In addition to the dark matter candidate, the model has a heavier neutral scalar and a charged scalar boson. These $Z_{2}$ odd particles can be probed directly at the LHC Run II [27-31] and the ILC [32,33]. The measurements of the branching fraction of the Higgs decay e.g., diphoton signal and invisible decay will be a probe of the $Z_{2}$ odd sector [33-36]. Also, there is a possibility of the inert doublet dark matter to be probed by indirect search [37-40]. Thus, the inert doublet model is well motivated dark matter model in both theoretical and phenomenological points of view.

The direct detection experiments give an important constraint on the inert doublet dark matter [5, 41, 42]. At the leading order, the inert doublet dark matter scatters with the quarks at the tree level, and with the gluon at the one-loop level by exchanging the SM Higgs boson. These contributions to the cross section for scattering of the dark matter on nucleon can be calculated in the same manner as the singlet scalar dark matter model [4345]. It is proportional to $\lambda_{A}^{2}$, where $\lambda_{A}$ is the effective Higgs-dark matter coupling which is defined in section 2. If $\lambda_{A}$ is not so small, they give dominant contribution to the cross section. However, if the dark matter mass $m_{A}$ is around a half of the SM Higgs boson mass, $\lambda_{A}$ should be suppressed because the SM Higgs boson $s$-channel exchange diagrams significantly contribute to the annihilation cross section which determines the relic amount of the dark matter. In this case, contributions which does not depends on $\lambda_{A}$ become important for the spin-independent cross section. For example, as shown in ref. [46], one-loop electroweak correction for the scattering with the light quarks gives an important correction.

In this paper, we revisit the radiative correction on the spin-independent cross section in the inert two-Higgs doublet model for the dark matter mass to be around a half of the Higgs boson mass. In particular, ref. [46] does not take into account for the effect of various scalar quartic couplings. We take into account for the non-zero values of the inert doublet couplings, which are equivalent to the mass difference between the dark matter and other $Z_{2}$ odd particles. They cannot be neglected in a viable parameter region 
in the light of the LEP II collider constraint [47, 48]. In addition to them, there is an interesting coupling, namely the self-coupling of the $Z_{2}$ odd particles, $\lambda_{2}$. This coupling is irrelevant for the phenomenology at the tree level, but we find it also plays the significant role here. Furthermore, we also evaluate contributions from twist-2 quark operators and two-loop diagrams of dark matter-gluon scattering. These contributions give the same order corrections as the scattering with quark at the one-loop level.

This paper is organized as follows. We briefly review the inert two-Higgs doublet model in section 2. In section 3, we review the calculation of the spin-independent cross section at the tree level, and introduce our strategy to incorporate the loop corrections to it. In section 4 , we show our result. We conclude in section 5. The details of the loop calculations are in the appendices.

\section{Model}

In this section, we briefly review the inert doublet model. In addition to the SM Higgs field $H$, we introduced a new $\mathrm{SU}(2)_{L}$ doublet scalar field $\Phi$ with $Y=1 / 2$. We impose $Z_{2}$ parity, under which the scalar fields behave as,

$$
H \rightarrow H, \quad \Phi \rightarrow-\Phi .
$$

Other quark and lepton fields are also invariant under the $Z_{2}$ parity as the SM Higgs field. Hence, $\Phi$ cannot have Yukawa interactions with the SM fermions. The generic potential of $H$ and $\Phi$ under the $Z_{2}$ parity is,

$$
\begin{aligned}
-V(H, \Phi)= & -m_{1}^{2} H^{\dagger} H-m_{2}^{2} \Phi^{\dagger} \Phi-\lambda_{1}\left(H^{\dagger} H\right)^{2}-\lambda_{2}\left(\Phi^{\dagger} \Phi\right)^{2} \\
& -\lambda_{3}\left(\Phi^{\dagger} \Phi\right)\left(H^{\dagger} H\right)-\lambda_{4}\left(\Phi^{\dagger} H\right)\left(H^{\dagger} \Phi\right)-\left(\frac{\lambda_{5}}{2}\left(\Phi^{\dagger} H\right)^{2}+\text { h.c. }\right) .
\end{aligned}
$$

We assume that $\Phi$ does not get any vacuum expectation value (VEV), then, the $Z_{2}$ parity which we have imposed is unbroken in the vacuum, and $m_{1}^{2}$ is related to the Higgs VEV and the coupling $\lambda_{1}$ as,

$$
m_{1}^{2}=-2 \lambda_{1} v^{2}
$$

where $v$ is the Higgs VEV, $v^{2}=\left(\sqrt{2} G_{F}\right)^{-1} \simeq(246 \mathrm{GeV})^{2} . G_{F}$ is the Fermi constant. Compared to the SM, we have additional five free parameters, $m_{2}^{2}, \lambda_{2}, \lambda_{3}, \lambda_{4}$ and $\lambda_{5}$. For the stability of this potential, the following relations are required [4]:

$$
\lambda_{1}>0, \quad \lambda_{2}>0, \quad \lambda_{3}>-2 \sqrt{\lambda_{1} \lambda_{2}}, \quad \lambda_{3}+\lambda_{4}-\left|\lambda_{5}\right|>-2 \sqrt{\lambda_{1} \lambda_{2}} .
$$

We can always take $\lambda_{5}$ as a real positive by a redefinition of the phase of $\Phi$ field. For example, when $\arg \lambda_{5}=\theta \neq 0$, we redefine $\Phi$ as $e^{i \theta / 2} \Phi$. Therefore, the inert doublet Higgs does not contribute to $C P$ violation. Hereafter we take a basis in which $\lambda_{5}$ is a real positive. In this basis, we parametrize the component fields of $H$ and $\Phi$ as follows,

$$
H=\left(\begin{array}{c}
-i \pi_{W}^{+} \\
\frac{v+h+i \pi_{Z}}{\sqrt{2}}
\end{array}\right), \quad \Phi=\left(\begin{array}{c}
-i H^{+} \\
\frac{S+i A}{\sqrt{2}}
\end{array}\right),
$$


where each component fields correspond to mass eigenstates. We can find mass eigenvalues of each particles and interaction terms. The mass eigenvalues are,

$$
\begin{aligned}
m_{h}^{2} & =2 v^{2} \lambda_{1}, \\
m_{H^{ \pm}}^{2} & =m_{2}^{2}+\frac{1}{2} \lambda_{3} v^{2}, \\
m_{S}^{2} & =m_{2}^{2}+\frac{1}{2}\left(\lambda_{3}+\lambda_{4}+\lambda_{5}\right) v^{2}, \\
m_{A}^{2} & =m_{2}^{2}+\frac{1}{2}\left(\lambda_{3}+\lambda_{4}-\lambda_{5}\right) v^{2} .
\end{aligned}
$$

As we mentioned in the above, we take $\lambda_{5}>0$ in this paper, hence $A$ is the lightest neutral $Z_{2}$ odd particle, and it is the dark matter candidate. ${ }^{1}$

The three-point interaction terms for the Higgs boson and the $Z_{2}$ odd particles are,

$$
\mathcal{L} \ni-\frac{1}{2}\left(\lambda_{3}+\lambda_{4}-\lambda_{5}\right) v h A^{2}-\lambda_{3} v h H^{+} H^{-}-\frac{1}{2}\left(\lambda_{3}+\lambda_{4}+\lambda_{5}\right) v h S^{2}
$$

The Higgs coupling to the dark matter is important to study dark matter phenomenology, and it is proportional to $\lambda_{3}+\lambda_{4}-\lambda_{5}$. So we denote it as

$$
\lambda_{A} \equiv \lambda_{3}+\lambda_{4}-\lambda_{5}
$$

We also introduce other short-handed notations,

$$
\begin{aligned}
\Delta m_{H^{ \pm}} & \equiv m_{H^{ \pm}}-m_{A}, \\
\Delta m_{S} & \equiv m_{S}-m_{A} .
\end{aligned}
$$

We treat $\left(m_{A}, \Delta m_{H^{ \pm}}, \Delta m_{S}, \lambda_{A}\right)$ as input parameters and determined $\left(m_{2}^{2}, \lambda_{3}, \lambda_{4}, \lambda_{5}\right)$ from these input parameters. Note that $\lambda_{2}$ is not related with these input parameters, and irrelevant for the analysis at tree level. However, $\lambda_{2}$ plays an important role at the loop level as we will see later. The loop correction to the dark matter mass is small for the light dark matter mass regime [51], so we keep using the above tree level relations among the mass and couplings in this paper.

In the following of this paper, we assume almost all of the energy density of the dark matter is comprised of the inert doublet dark matter which is generated as a thermal relic. The amount of thermal relic is controlled by the annihilation cross section of the dark matter [6-8]. There are some comprehensive studies on viable parameter regions [42, 4953]. Because of its $\mathrm{SU}(2)_{L}$ charge, $A A \rightarrow W W^{(*)}$ channel gives a significant contribution to the annihilation cross section for the case of $m_{A} \gtrsim m_{W}$ [54], and it tends to be too large to obtain the correct abundance $\Omega_{\mathrm{DM}} h^{2}=0.1196 \pm 0.0031$ [55]. It is known that there are two parameter regions to obtain the correct relic abundance $[52,53]$. One region is the light mass region with $m_{A} \lesssim 72 \mathrm{GeV}$, in which $A A \rightarrow W W^{*}$ becomes less significant because it is well below energy threshold of two body $W W$ mode. The other region is the heavy

\footnotetext{
${ }^{1}$ Some references assume $S$ is the lightest $Z_{2}$ odd particle. However, this is just a difference of the basis of $\Phi$. For example, if we define $\Phi^{\prime} \equiv i \Phi$, we can see $S^{\prime}=-A$ and $A^{\prime}=S$. Hence, there is no physical difference.
} 
mass region with $m_{A} \gtrsim 600 \mathrm{GeV}$, in which the annihilation cross section is suppressed by its mass. ${ }^{2}$

Since the inert doublet dark matter couples with the SM Higgs field via the coupling $\lambda_{A}$, the dark matter can scatter with nucleus and the direct detection experiment gives an important constraint on the coupling $\lambda_{A}[5,41,42]$. Especially, this constraint gives a large impact on the light mass region. This is because the amount of the relic abundance is also controlled by the same coupling. As a result, the region with $m_{A} \lesssim 53 \mathrm{GeV}$ is already excluded by the LUX experiment, and viable region in the light mass range is $53 \mathrm{GeV} \lesssim m_{A} \lesssim 72 \mathrm{GeV}[52,53]$. In this viable range, although the coupling $\lambda_{A}$ is small, the annihilation cross section is enhanced because of the propagator of the SM Higgs boson in $s$-channel. However, the scattering of a nucleon and a dark matter does not hit the SM Higgs pole, and thus the spin-independent cross section is just suppressed by the coupling $\lambda_{A}$. Therefore the contributions which is independent of $\lambda_{A}$, i.e., the radiative corrections on the spin-independent cross section becomes important in this mass range.

\section{Spin-independent cross section}

In this section, we formulate how to include radiative corrections to the spin-independent cross section. To calculate the cross section of elastic scattering of dark matter and nucleon, first, we construct the effective interaction of the dark matter and quark/gluon. The relevant terms for our calculation are written as,

$$
\begin{aligned}
\mathcal{L}_{\text {eff. }}= & \frac{1}{2} \sum_{q=u, d, s} \Gamma^{q} A^{2}\left(m_{q} \bar{q} q\right)-\frac{1}{2} \frac{\alpha_{s}}{4 \pi} \Gamma^{G} A^{2} G_{\mu \nu}^{a} G^{a \mu \nu} \\
& +\frac{1}{2 m_{A}^{2}} \sum_{q=u, d, s, c, b}\left[\left(\partial^{\mu} A\right)\left(\partial^{\nu} A\right) \Gamma_{\mathrm{t} 2}^{q} \mathcal{O}_{\mu \nu}^{q}-A\left(\partial^{\mu} \partial^{\nu} A\right) \Gamma_{\mathrm{t} 2}^{\prime q} \mathcal{O}_{\mu \nu}^{q}\right],
\end{aligned}
$$

where $\mathcal{O}_{\mu \nu}^{q}$ is the quark twist-2 operator which is defined as,

$$
\mathcal{O}_{\mu \nu}^{q} \equiv \frac{i}{2} \bar{q}\left(\partial_{\mu} \gamma_{\nu}+\partial_{\nu} \gamma_{\mu}-\frac{1}{2} g_{\mu \nu} \not \partial\right) q
$$

In the effective Lagrangian given in eq. (3.1), we neglect higher twist gluon operators because their contributions are suppressed by $\alpha_{s}$ compared to the twist-0 gluon operator [57]. The coefficients $\Gamma$ are determined by matching with UV Lagrangian, which will be explained later. To calculate the scattering amplitude of nucleon, we also need matrix elements of quark/gluon operators, which are given as,

$$
\begin{aligned}
\left\langle N\left|m_{q} \bar{q} q\right| N\right\rangle & =m_{N} f_{q}, \\
-\frac{9 \alpha_{s}}{8 \pi}\left\langle N\left|G_{\mu \nu}^{a} G^{a \mu \nu}\right| N\right\rangle & =m_{N} f_{g}, \\
\left\langle N\left|\mathcal{O}_{\mu \nu}^{q}\right| N\right\rangle & =\frac{1}{m_{N}}\left(p_{\mu} p_{\nu}-\frac{1}{4} m_{N}^{2} g_{\mu \nu}\right)(q(2)+\bar{q}(2)) .
\end{aligned}
$$

\footnotetext{
${ }^{2}$ Ref. [56] pointed out another parameter region in which some of diagrams of $A A \rightarrow W W$ cancel out. However, this parameter region is severely constrained by the LUX experiment. See, ref. [52].
} 


\begin{tabular}{|c|c|c|c|c|c|}
\hline$f_{u}$ & 0.0110 \\
$f_{d}$ & 0.0273 \\
$f_{s}$ & 0.0447 \\
\hline
\end{tabular}$\quad$\begin{tabular}{cc|c|c|}
\hline$u(2)$ & 0.11 & $\bar{u}(2)$ & 0.036 \\
$d(2)$ & 0.22 & $\bar{d}(2)$ & 0.034 \\
$s(2)$ & 0.026 & $\bar{s}(2)$ & 0.026 \\
$c(2)$ & 0.019 & $\bar{c}(2)$ & 0.019 \\
$b(2)$ & 0.012 & $\bar{b}(2)$ & 0.012 \\
\hline
\end{tabular}

Table 1. Matrix elements for neutron. Left panel shows the matrix elements for quark twist-0 operators, which are taken from the default values of micrOMEGAs [62]. Right panel shows the second moments for quark distribution function, which are evaluated at the scale of $\mu=m_{Z}$ by using the CTEQ parton distribution functions [60].

$f_{g}$ is related to $f_{q}$ as,

$$
f_{g}=1-\sum_{q=u, d, s} f_{q}
$$

This relation is derived by using the relation obtained from the trace anomaly [58],

$$
m_{N}=\left\langle N\left|T_{\mu}^{\mu}\right| N\right\rangle=-\frac{9 \alpha_{s}}{8 \pi}\left\langle N\left|G_{\mu \nu}^{a} G^{a \mu \nu}\right| N\right\rangle+\sum_{q=u, d, s}\left\langle N\left|m_{q} \bar{q} q\right| N\right\rangle .
$$

From this discussion, we can see $\left\langle N\left|m_{q} \bar{q} q\right| N\right\rangle$ and $\left(\alpha_{s} / 4 \pi\right)\left\langle N\left|G_{\mu \nu}^{a} G^{a \mu \nu}\right| N\right\rangle$ are same order. Thus, the calculation at the $n$-loop order requires the $(n+1)$-loop order calculation for diagrams with $G_{\mu \nu}^{a} G^{a \mu \nu}$. For $q(2)$ and $\bar{q}(2)$, we can see that they are the second moments of the quark and anti-quark parton distribution functions by using a discussion of operator product expansion as, ${ }^{3}$

$$
q(2)+\bar{q}(2)=\int_{0}^{1} d x(q(x)+\bar{q}(x))
$$

We use the CTEQ parton distribution functions [60] to evaluate them, and use the same value used in [61].

We have checked that the spin-independent cross section of a dark matter and a proton is the almost same as of the a dark matter and a neutron. Their difference is smaller than a few percent in almost all of the parameter region. In the following of this paper, we calculate the scattering cross section of a dark matter and a neutron. The matrix elements which are used are summarized in table 1. By using the above matrix elements and the coefficients $\Gamma$ 's in the effective interaction given in eq. (3.1), the scattering amplitude of the nucleon and the dark matter is given as,

$$
\begin{aligned}
i \mathcal{M} & =i m_{N}\left[\sum_{q} \Gamma^{q} f_{q}+\frac{2}{9} \Gamma^{G} f_{g}+\frac{3}{4} \sum_{q}\left(\Gamma_{\mathrm{t} 2}^{q}+\Gamma_{\mathrm{t} 2}^{\prime q}\right)(q(2)+\bar{q}(2))\right], \\
\sigma_{\mathrm{SI}} & =\frac{\mu^{2}}{4 \pi m_{A}^{2}}|\mathcal{M}|^{2},
\end{aligned}
$$

where $\mu$ is the reduced mass, which is defined as $\mu \equiv m_{N} m_{A} /\left(m_{N}+m_{A}\right)$. Hence, what we have to calculate is the effective coupling $\Gamma$ 's.

\footnotetext{
${ }^{3}$ For example, see section 18.5 in Peskin-Schroeder's textbook [59].
} 




(a)

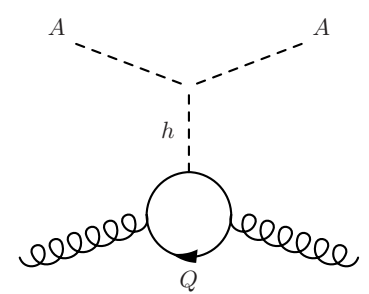

(b)

Figure 1. The diagrams which contribute to the spin-independent cross section at the leading order.

\subsection{At the leading order}

We start to give a brief review on the calculation at the leading order. We need to calculate the elastic scattering cross section for the dark matter and nucleon system, $\sigma(\mathrm{DM} N \rightarrow$ DM $N$ ), where $N$ stands for the nucleon. As described before, we construct the effective Lagrangian with the gluon and the light quarks $q=u, d, s$ by integrating out the heavy quarks $Q=c, b, t$ and the SM Higgs boson. We should take into account the one-loop diagrams for the scattering with gluon, because their contributions are same order as the tree-level scattering with the light quarks. The dark matter scatters with the SM quarks at the tree level and the gluon at the one-loop level as shown in figure 1(a) and 1(b), respectively. Their amplitudes are proportional to the effective Higgs-dark matter coupling $\lambda_{A}$. From these processes, the following relevant operators for the spin-independent cross section are generated,

$$
A^{2} \bar{q} q, \quad A^{2} G_{\mu \nu}^{a} G^{a \mu \nu} .
$$

The coefficients of the effective Lagrangian given at the leading order is determined as,

$$
\Gamma^{q}=\Gamma^{G}=\frac{\lambda_{A}}{m_{h}^{2}}, \quad \Gamma_{\mathrm{t} 2}^{q}=\Gamma_{\mathrm{t} 2}^{\prime q}=0 .
$$

Using these coefficients and eq. (3.10), we can calculate the amplitude of the process and the spin-independent cross section as,

$$
\sigma_{\mathrm{SI}}=\frac{1}{4 \pi} \frac{\lambda_{A}^{2} \mu^{2} m_{N}^{2} f_{N}^{2}}{m_{A}^{2} m_{h}^{4}}
$$

where,

$$
f_{N} \equiv \frac{2}{9}+\frac{7}{9} \sum_{q} f_{q} .
$$

\subsection{At the next leading order}

We move to calculate the loop corrections to the spin-independent cross section. We need to consider the loop corrections to the four relevant operators for the spin-independent cross section,

$$
A^{2} \bar{q} q, \quad A^{2} G_{\mu \nu}^{a} G^{a \mu \nu}, \quad\left(\partial^{\mu} A\right)\left(\partial^{\nu} A\right) \mathcal{O}_{\mu \nu}^{q}, \quad A\left(\partial^{\mu} \partial^{\nu} A\right) \mathcal{O}_{\mu \nu}^{q}
$$




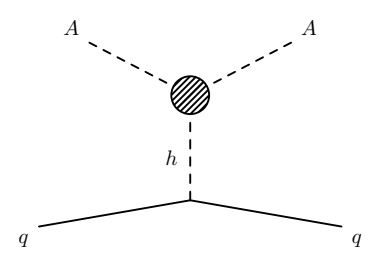

(a)

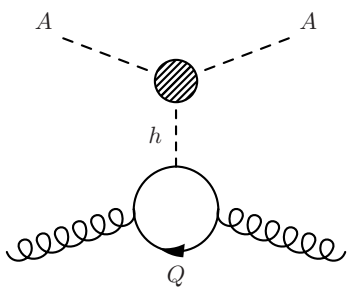

(d)

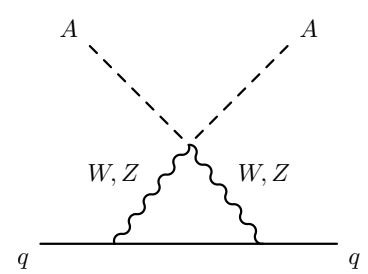

(b)

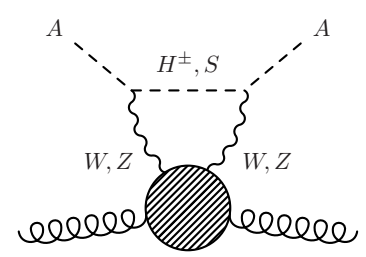

(e)

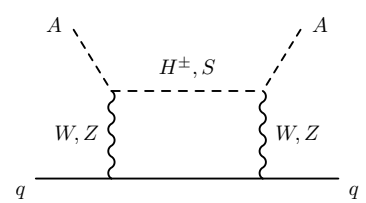

(c)



(f)

Figure 2. The diagrams we calculate. The shaded region is one-loop correction.

There are some remarks on this calculation. First, trace anomaly relation eq. (3.7) is suffered from QCD correction at the next-leading order. However, we consider $\lambda_{A}$ is not so large, and assume corrections of the order of $\lambda_{A} \alpha_{s} / 4 \pi$ can be neglected. Also, for the contribution which is independent of $\lambda_{A}$, we only take into account the leading order of $\alpha_{s}$. Thus, for the scattering with the gluon, we can still use eq. (3.7) even in the loop level calculation. Second, we evaluate the effect of twist-2 operator $\mathcal{O}_{\mu \nu}^{q}$ at the scale $\mu=m_{Z}$. Thus, we take into account $q=u, d, s, c$ and $b$ and evaluate the matrix element of $\mathcal{O}_{\mu \nu}^{q}$ by using the parton distribution functions at $\mu=m_{Z}$.

The diagrams we need to calculate are shown in figure 2. The diagrams with gluons are two-loop diagrams but contribute to the spin-independent cross section as the one-loop order correction as we mentioned in section 3.1. There are some diagrams which are the same order but not shown in figure 2. They are proportional to the Higgs coupling to the dark matter, $\lambda_{A}$. We are interested in the case that this coupling is very small. Thus the diagrams with this coupling give much smaller contributions than the diagrams shown in figure 2, and do not need to be calculated. Here we parametrized the loop corrections to the $\lambda_{A}$ as $\delta \Gamma_{h}\left(q_{h}^{2}\right)$, and denote the correction from the box and triangle diagrams as $\Gamma_{\text {Box }}^{q}$. Here $q_{h}^{2}$ is the momentum squared of the Higgs boson. What we need is the scattering amplitude in the non-relativistic limit. In the limit of zero momentum transfer, the amplitudes of the diagrams given in figure 2 are written as,

$$
\text { Figure } 2(a)=\frac{i \delta \Gamma_{h}(0)}{m_{h}^{2}} m_{q} \bar{u} u,
$$

Figure $2(b)+$ Figure $2(c)=i \Gamma_{\mathrm{Box}}^{q} m_{q} \bar{u} u+\frac{i}{m_{A}^{2}}\left(\Gamma_{\mathrm{t} 2}^{q}+\Gamma_{\mathrm{t} 2}^{\prime q}\right) \bar{u}\left((p q) p-\frac{1}{4} p^{2} \not q\right) u$,

$$
\text { Figure } 2(d)=\frac{i \delta \Gamma_{h}(0)}{m_{h}^{2}} \times \frac{2}{9}\left(-\frac{9 \alpha_{s}}{8 \pi} G_{\mu \nu}^{a} G^{a \mu \nu}\right)
$$

Figure $2(e)+$ Figure $2(f)=i \Gamma_{\text {Box }}^{G} \times \frac{2}{9}\left(-\frac{9 \alpha_{s}}{8 \pi} G_{\mu \nu}^{a} G^{a \mu \nu}\right)$. 
In eq. (3.17), $p^{\mu}$ and $q^{\mu}$ is momentum of the dark matter and quark, respectively. We have used equation of motion of quark, $\not q u=m_{q} u . \Gamma_{\mathrm{t} 2}^{q}+\Gamma_{\mathrm{t} 2}^{q}$ can be read from the above amplitudes, and $\Gamma^{q}$ and $\Gamma^{G}$ is determined as,

$$
\Gamma^{q}=\frac{\delta \Gamma_{h}(0)}{m_{h}^{2}}+\Gamma_{\mathrm{Box}}^{q}, \quad \Gamma^{G}=\frac{\delta \Gamma_{h}(0)}{m_{h}^{2}}+\Gamma_{\mathrm{Box}}^{g} .
$$

Here we treat the gluon field as the background field and neglect its higher twist operators. For the detail of the calculation of $\Gamma$ 's, see the appendices.

We need to discuss how to calculate the value of $\lambda_{A}$ and renormalization condition. In the tree level calculation, we set this coupling to reproduce the current relic abundance of the dark matter in our universe. Now we need to take into account the one-loop effect. Since our focus is $m_{A} \simeq m_{h} / 2$ regime, the dominant contribution for the relic abundance calculation is coming from the diagram shown in figure 3 because this diagram picks up the Higgs resonance. Hence it is only the vertex correction that we should take into account, and we can ignore other one-loop corrections, such as box diagrams, in the relic abundance calculation. Therefore we can set $\lambda_{A}$ by the following relation,

$$
\left|\lambda_{A}+\delta \Gamma_{h}\left(m_{h}^{2}\right)+\delta_{\lambda_{A}}\right|^{2}=\left|\lambda_{\text {relic }}\right|^{2},
$$

where $\delta_{\lambda_{A}}$ is the counter-term. $\lambda_{\text {relic }}$ is the effective Higgs boson coupling to the dark matter, and is determined as to reproduce the correct relic abundance. Since the annihilation cross section determine the relic abundance, the square of the couplings appear in the relation above. Thus, we have two solution for $\lambda_{A}$,

$$
\lambda_{A}= \pm\left|\lambda_{\text {relic }}\right|-\delta \Gamma_{h}\left(m_{h}^{2}\right)-\delta_{\lambda_{A}} .
$$

This is crucial in $\sigma_{\mathrm{SI}}$ calculation at the loop level because there is interference between the tree and the loop diagrams as we can see in eq. (3.10). Depending on the sign in eq. (3.22), the interference is destructive or constructive, and we find two solutions for $\sigma_{\mathrm{SI}}$. This point was overlooked in ref. [46]. Now the value of $\lambda_{A}$ is set by eq. (3.22). It is useful to renormalize $\lambda_{A}$ to make that $\delta \Gamma_{h}\left(m_{h}^{2}\right)=-\delta_{\lambda_{A}}$ is satisfied. By using this condition, we can take $\lambda_{A}$ as $\pm\left|\lambda_{\text {relic }}\right|$.

We would like to mention on the stability condition here. Since $\lambda_{A}= \pm\left|\lambda_{\text {relic }}\right|$, there are two parameter sets for $\left(\lambda_{3}, \lambda_{4}, \lambda_{5}\right)$ for each $\lambda_{A}$. These parameter sets have to satisfy the stability condition given in eq. (2.4). For $53 \mathrm{GeV}<m_{\mathrm{DM}}<71 \mathrm{GeV}, 100 \mathrm{GeV}<m_{S}<$ $250 \mathrm{GeV}$, and $100 \mathrm{GeV}<m_{H^{ \pm}}<250 \mathrm{GeV}$, we find the first three conditions in eq. (2.4) are always satisfied, and the last one is satisfied if $\lambda_{2} \gtrsim 0.001$. This constraint on $\lambda_{2}$ is very weak and almost harmless.

It is useful to define "effective coupling" $\lambda_{A}^{\text {eff. }} \equiv \lambda_{A}+\delta \lambda$ which is relevant for $\sigma_{\mathrm{SI}}$, where $\delta \lambda$ is defined as,

$$
\delta \lambda \equiv \delta \Gamma_{h}(0)+\delta_{\lambda_{A}}+\frac{m_{h}^{2}}{f_{N}}\left(\sum_{q} \Gamma_{\mathrm{Box}}^{q} f_{q}\right)+\frac{2}{9} \frac{m_{h}^{2}}{f_{N}} \Gamma_{\mathrm{Box}}^{G} f_{g}+\frac{3}{4} \frac{m_{h}^{2}}{f_{N}} \sum_{q}\left(\Gamma_{\mathrm{t} 2}^{q}+\Gamma_{\mathrm{t} 2}^{q}\right)(q(2)+\bar{q}(2)) .
$$






Figure 3. The diagram giving the dominant contribution in the relic abundance calculation for $m_{\mathrm{DM}} \simeq m_{h} / 2$. The shaded region contains tree and loop corrections. Other diagrams, such as box diagrams, give a small correction to this diagrams for $m_{\mathrm{DM}} \simeq m_{h} / 2$.

Note that we determined $\delta_{\lambda_{A}}=-\delta \Gamma_{h}\left(m_{h}^{2}\right)$ in the previous paragraph. By using $\lambda_{A}^{\text {eff. }} \equiv$ $\lambda_{A}+\delta \lambda$, the spin-independent cross section at the next-leading order is written in the similar way as the tree level formula eq. (3.13),

$$
\sigma_{\mathrm{SI}}=\frac{1}{4 \pi} \frac{\left(\lambda_{A}^{\text {eff. }}\right)^{2} \mu^{2} m_{N}^{2} f_{N}^{2}}{m_{A}^{2} m_{h}^{4}}=\frac{1}{4 \pi} \frac{\left( \pm\left|\lambda_{\text {relic }}\right|+\delta \lambda\right)^{2} \mu^{2} m_{N}^{2} f_{N}^{2}}{m_{A}^{2} m_{h}^{4}} .
$$

In the next section, we show our numerical results by using the relation we find in this section. The analytic expressions and the details of the calculation are in the appendix.

When $m_{\mathrm{DM}}>m_{h} / 2$, it is kinematically forbidden to hit the pole of the Higgs propagator, and the enhancement of the cross section due to the Higgs resonance does not happen. The dominant contribution to the dark matter annihilation cross section does not come from $\sqrt{s}=m_{h}^{2}$ but from $\sqrt{s} \simeq 4 m_{\mathrm{DM}}^{2}>m_{h}^{2}$ for $m_{\mathrm{DM}}>m_{h} / 2$. Therefore we replace $\delta \Gamma_{h}\left(m_{h}^{2}\right)$ in the above equations into $\delta \Gamma_{h}\left(4 m_{\mathrm{DM}}^{2}\right)$ for $m_{\mathrm{DM}}>m_{h} / 2$.

\section{Results}

We start by showing the tree level result on $\lambda_{A}$ to find the mass region in which the loop correction becomes significant. In figure 4 , we show the absolute value of the Higgs boson coupling to the dark matter, $\lambda_{A}$ at the tree level as a function of the dark matter mass. This coupling is determined by requiring to reproduce the current relic abundance of the dark matter in our universe, and is the same as $\left|\lambda_{\text {relic }}\right|$ defined in eq. (3.21). It is calculated by using micrOMEGAs [62]. Since we are interested in the small coupling regime, we focus on $53 \mathrm{GeV}<m_{\mathrm{DM}}<64 \mathrm{GeV}$. In this plot, we take $\Delta m_{H^{ \pm}}=\Delta m_{S}=50 \mathrm{GeV}$, but these parameter dependence is very week as long as the mass difference is large enough to ignore the co-annihilation process, namely $\Delta m_{S, H^{ \pm}} \gtrsim 20 \mathrm{GeV}$.

We move to discuss on the effect of the loop correction. We show the value of $\delta \lambda$ for $\Delta m_{H^{ \pm}}=\Delta m_{S}=50 \mathrm{GeV}$ in figure 5. The three lines correspond to the different $\lambda_{2}$ choices. We find $\delta \lambda$ is the order of $10^{-3}$. Thus, the radiative correction becomes important for $\left|\lambda_{\text {relic }}\right| \lesssim \mathcal{O}\left(10^{-3}\right)$, namely $55 \mathrm{GeV} \lesssim m_{\mathrm{DM}} \lesssim 63 \mathrm{GeV}$, where the tree level coupling is comparable or even smaller than the one-loop level value as we can see from figure 4 .

Now $\delta \lambda$ depends on the four parameters, $\lambda_{2}, m_{\mathrm{DM}}, \Delta m_{H^{ \pm}}, \Delta m_{S}$. We show these parameter dependence of $\delta \lambda$ in figure 6 . Here we take $m_{H^{ \pm}}=m_{S}$. This parameter choice enhances the custodial symmetry in $Z_{2}$ odd sector and suppress the contributions to the $T$ 


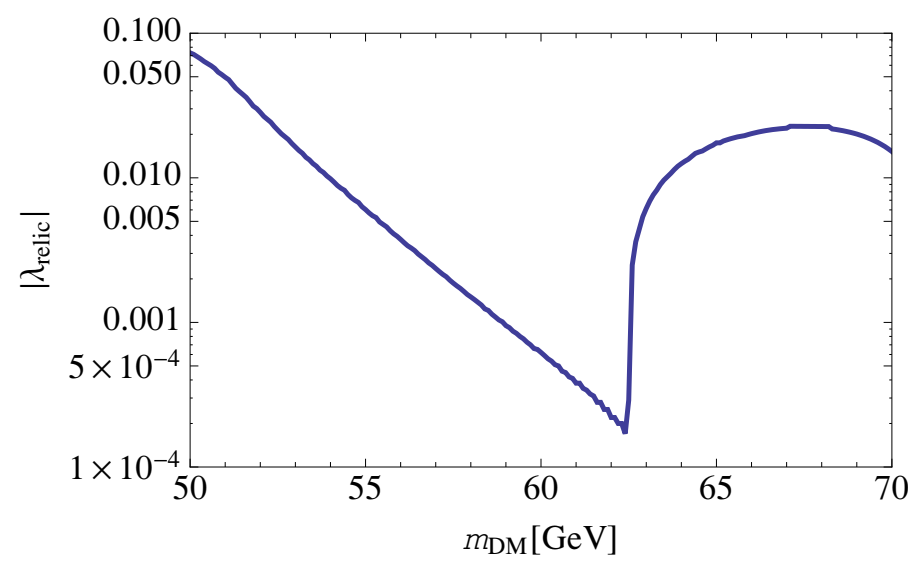

Figure 4. The absolute value of the effective couplings as a function of the dark matter mass for $m_{H^{ \pm}}=m_{S}=m_{D M}+50 \mathrm{GeV}$. This coupling is determined so as to reproduce the correct relic abundance, and is the same as the $\lambda_{A}$ determined at the tree level analysis.



Figure 5. The value of $\delta \lambda$ defined in eq. (3.23). The red, blue, and black lines are for $\lambda_{2}=0,0.5$, and 1.0, respectively. Here we fixed $m_{H^{ \pm}}-m_{D M}=m_{S}-m_{D M}=50 \mathrm{GeV}$.

parameter from $Z_{2}$ odd sector. We find that $\delta \lambda$ weakly depends on $m_{\mathrm{DM}}$, and is sensitive to the value of $\Delta m_{S, H^{ \pm}}$and $\lambda_{2}$. The dependence on $\Delta m_{S, H^{ \pm}}$is contrast to the tree level analysis where $\left|\lambda_{\text {relic }}\right|$ is almost independent from $\Delta m_{S, H^{ \pm}}$as long as $\Delta m_{S, H^{ \pm}} \gtrsim 20 \mathrm{GeV}$. Another feature is the larger $\lambda_{2}$ makes $\delta \lambda$ to be zero. This means the terms proportional to $\lambda_{2}$ cancel the other loop contributions.

We show the spin-independent cross section both at the tree and loop levels as a function of the dark matter mass in figure 7, with the current bound [63] and future prospects [64-66]. The value of $\lambda_{2}$ is different in each panels. We take $\Delta m_{H^{ \pm}}=\Delta m_{S}=$ $50 \mathrm{GeV}$ as a benchmark. Since the sign of the tree level coupling, $\lambda_{A}$, is unknown, there are two possibilities for the result at the loop level. The feature is highly depend on the sign of $\lambda_{A}$, and we see that the spin-independent cross section at the loop level is both larger and smaller than the one at the tree level value. For large $\lambda_{2}$ region, the sign of the loop correction to the effective coupling is flipped as we can see from the upper-left and lower-right panels. In this benchmark, the loop corrections vanish when $\lambda_{2} \simeq 1.45$ because the loop corrections depending on $\lambda_{2}$ cancel the other loop corrections. From the figure, 

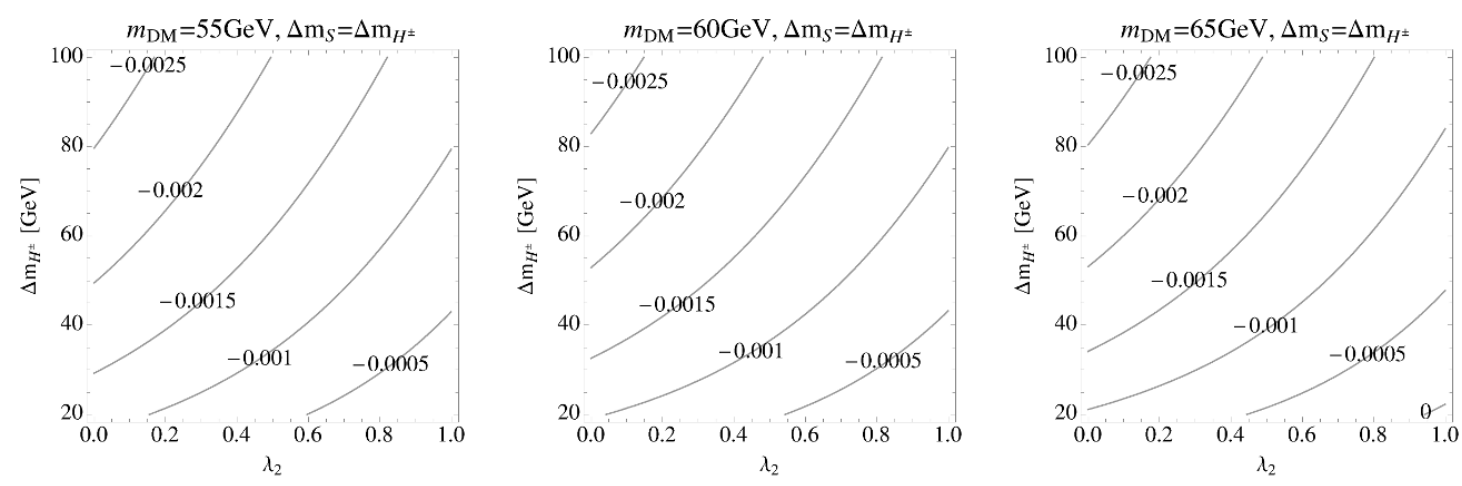

Figure 6. The value of $\delta \lambda$ as a function of $\lambda_{2}$ and mass difference between the dark matter and other $Z_{2}$ odd particles. Here we take $m_{H^{ \pm}}=m_{S}$. The dark matter mass of each panels are $m_{D M}=55 \mathrm{GeV}$ (left), $60 \mathrm{GeV}$ (middle), and $65 \mathrm{GeV}$ (right).

we can see the importance of the loop corrections in this dark matter mass region. For $\lambda_{2}=0.3$ case, for example, we have a chance to detect $62 \mathrm{GeV}$ dark matter in the future, although it is impossible according to the tree level analysis. On the other hand, it might be impossible for $\sim 58 \mathrm{GeV}$ dark matter to be detected, although it is possible according to the tree level analysis. Thus the detectable dark matter mass range is modified due to the loop correction, and it is also depend on the model parameters, especially the dark matter self-interacting coupling $\lambda_{2}$. Since we do not know the value of $\lambda_{2}$, we can not give a strict prediction on the spin-independent cross section in this dark matter mass region. We varied the value of $\lambda_{2}$ for $0<\lambda_{2}<1.45$, where the perturbative calculation works well, and make a plot in figure 8. The yellow region is the model prediction for $\Delta m_{S}=\Delta m_{H^{ \pm}}=50 \mathrm{GeV}$.

So far we have chosen $\Delta m_{S}=\Delta m_{H^{ \pm}}=50 \mathrm{GeV}$. However, the choice of these mass difference also play the significant role for $\sigma_{\mathrm{SI}}$ as we can see from figure 6 . In this paragraph, we vary these parameter keeping the custodial symmetric limit, $\Delta m_{S}=\Delta m_{H^{ \pm}}$. We make plots the $\sigma_{\mathrm{SI}}$ in $\left(m_{\mathrm{DM}}, \lambda_{2}\right)$-plain in figure 9 , and in $\left(m_{\mathrm{DM}}, m_{H^{ \pm}}\right)$-plain in figure 10 . The red region is basically beyond the discovery limit caused by atmospheric and astrophysical neutrinos, and we can see that the dark matter mass range in which the dark matter is possible to be detected in the future direct detection experiments is highly depending on the model parameter.

Finally, we give an approximate formula for $\delta \lambda$ which is defined in eq. (3.23). In the case of $m_{H}^{ \pm}=m_{S}$,

$$
\begin{aligned}
\delta \lambda= & -0.00409 m_{\mathrm{DM}}\left(0.0000144-7.77 \times 10^{-8} m_{H^{ \pm}}-0.00334 \frac{1}{m_{H^{ \pm}}}\right) \\
& +\lambda_{2}\left(0.00183-7.87 \times 10^{-10} m_{H^{ \pm}}^{2}+m_{\mathrm{DM}}^{2}\left(-4.13 \times 10^{-8}-\frac{0.00113}{m_{H^{ \pm}}^{2}}\right)\right) .
\end{aligned}
$$

By using the above expression and eq. (3.24), an approximate value of the cross section can be obtained. We have checked its error is less than $2 \%$ in the range of $50<m_{\mathrm{DM}}<62.5 \mathrm{GeV}$ and $100<m_{H^{ \pm}}=m_{S}<250 \mathrm{GeV}$. 

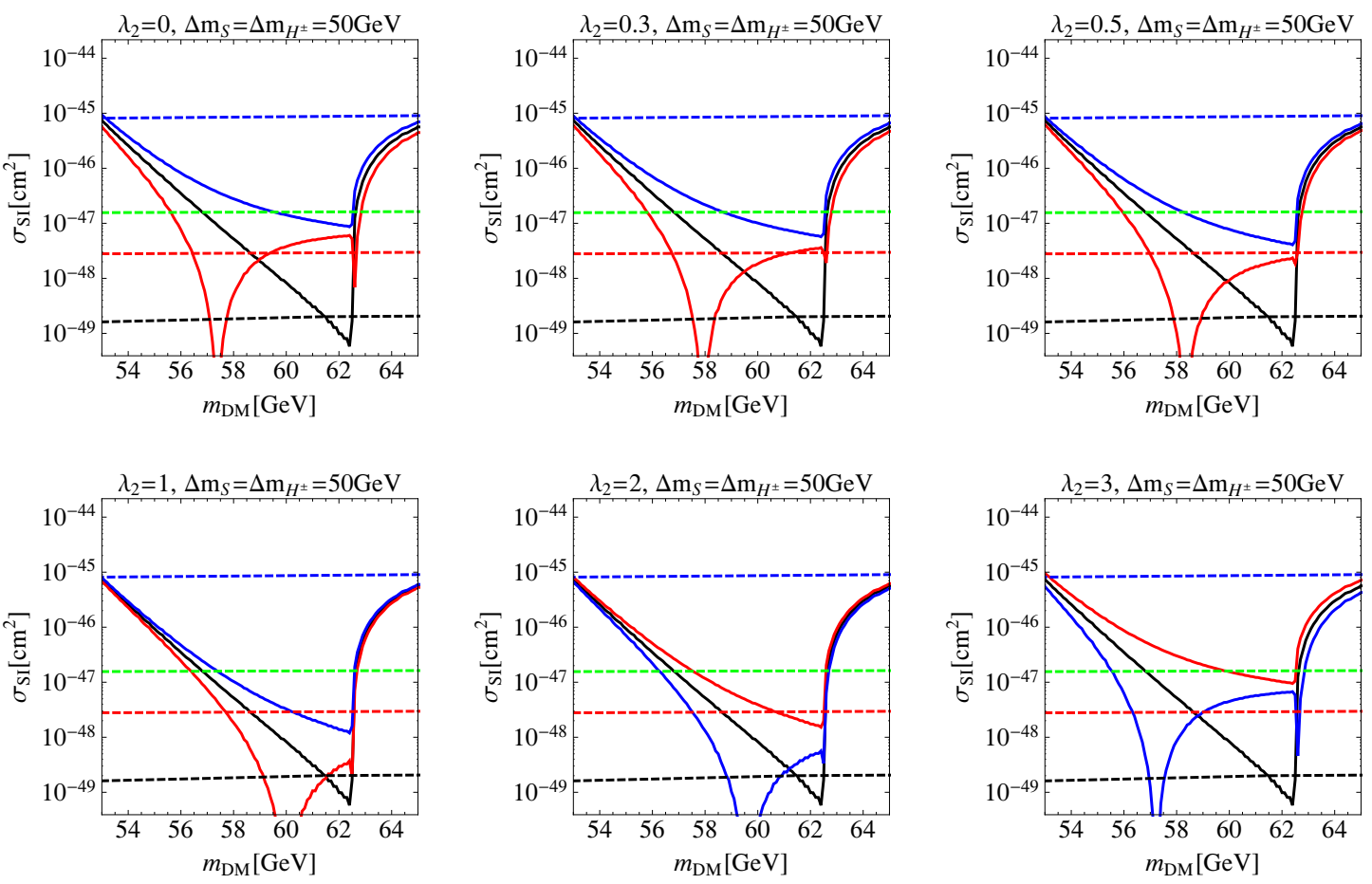

Figure 7. The spin-independent cross section at tree level (black-solid line), and loop level (redsolid and blue-solid lines). Since the sign of the tree level coupling, $\lambda_{\text {relic }}$, is unknown, there are two possibility for the result at loop level. If the couplings at tree and loop levels are constructive (destructive), the effective coupling is blue (red) line. Here $\lambda_{2}=0$ (upper-left), $\lambda_{2}=0.3$ (uppermiddle), $\lambda_{2}=0.5$ (upper-right), $\lambda_{2}=1.0$ (lower-left), $\lambda_{2}=2.0$ (lower-middle), and $\lambda_{2}=3.0$ (lower-right). The current bound and future prospects are also shown. The blue-dashed line is the current LUX bound. The green-dashed, red-dashed lines are the future prospect by XENON1T and LZ, respectively, and the black-dashed line is the discovery limit caused by atmospheric and astrophysical neutrinos.

\section{Conclusion and discussion}

In this paper, we discussed the spin-independent cross section $\sigma_{\mathrm{SI}}$ of nucleon and the dark matter in the inert doublet model. We revisited the radiative corrections to the spinindependent cross section with taking into account the effect of the non-zero values of the inert doublet couplings, namely the mass differences among $Z_{2}$ odd particles and the dark matter self coupling $\lambda_{2}$. The effect of these couplings were ignored in the previous work [46], but we find they actually control the main contribution in the radiative corrections.

The sign of the tree level coupling is important for precise prediction of the spinindependent cross section. Depending on its sign, the spin-independent cross section at the one-loop level becomes bigger or smaller than the tree level prediction. When it becomes bigger, the direct detect experiments have chance to detect the dark matter even if its mass is a half of the Higgs mass. This feature can not found at the tree level analysis.

The unknown model parameters are the origin of the uncertainty for the model prediction to the spin-independent cross section. Once the LHC experiment find the extra scalars, $S$ and $H^{ \pm}$, and determined their masses, the uncertainty will be reduced. 




Figure 8. The spin-independent cross section at tree level (black-solid line), and loop level (yellow shaded region). Here we vary $\lambda_{2}$ for $0<\lambda_{2}<1.45$. The blue-dashed line is the current LUX bound. The green-dashed, red-dashed lines are the future prospect by XENON1T and LZ, respectively, and the black-dashed line is the discovery limit caused by atmospheric and astrophysical neutrinos. Here we take $\Delta m_{H^{ \pm}}=50 \mathrm{GeV}, \Delta m_{S}=50 \mathrm{GeV}$.

\section{Acknowledgments}

The authors thank Natsumi Nagata for useful discussion. The work is supported by MEXT Grant-in-Aid for Scientific Research on Innovative Areas (No. 23104006 [TA]) and JSPS Research Fellowships for Young Scientists [RS].

\section{A Shorthand notations}

In the appendices, we give explicit formulae for the loop corrections to the spin-independent cross section. Electroweak gauge couplings are defined as,

$$
g_{W}=\frac{e}{s}, \quad g_{Z}=\frac{e}{s c}, \quad g_{f_{L}}=g_{Z}\left(T_{3, f}-s^{2} Q_{f}\right), \quad g_{f_{R}}=-g_{Z} s^{2} Q_{f},
$$

where $f$ runs through $u, d, s, c, b$ and $t$.

\section{B One-loop box type diagrams}

We calculate one-loop box diagrams which contribute to the $q A \rightarrow q A$ process. We consider only the light quarks. We expand the diagrams by the masses of the light quarks and keep only its leading order. This calculation is for the spin-independent cross section, and we can assume the momentum transfer is small, we take it zero. The sum of the diagrams we calculate in this section give the contributions to $\Gamma_{\mathrm{Box}}^{q}, \Gamma_{t 2}^{q}$, and $\Gamma_{t 2}^{\prime q}$ through,

$$
i \Gamma_{\mathrm{Box}}^{q} m_{q}+\frac{i}{m_{A}^{2}}\left(\Gamma_{t 2}^{q}+\Gamma_{\mathrm{t} 2}^{q}\right)\left(p^{\mu} q_{\mu} \not p-\frac{1}{4} p^{2} \not q\right) .
$$

The definitions of $\Gamma_{\mathrm{Box}}^{q}, \Gamma_{t 2}^{q}$, and $\Gamma_{t 2}^{q}$ are given in eq. (3.1). 



Figure 9. The $\sigma_{\mathrm{SI}}$ in $\left(m_{\mathrm{DM}}, \lambda_{2}\right)$-plain. The value of $\sigma_{\mathrm{SI}}$ is $\sigma_{\mathrm{SI}}<10^{-49} \mathrm{~cm}^{2}, 10^{-49} \mathrm{~cm}^{2}<\sigma_{\mathrm{SI}}<$ $10^{-48} \mathrm{~cm}^{2}, 10^{-48} \mathrm{~cm}^{2}<\sigma_{\mathrm{SI}}<10^{-47} \mathrm{~cm}^{2}, 10^{-47} \mathrm{~cm}^{2}<\sigma_{\mathrm{SI}}<10^{-46} \mathrm{~cm}^{2}$, and $10^{-46} \mathrm{~cm}^{2}<\sigma_{\mathrm{SI}}$ in the red, orange, yellow, green, and cyan regions, respectively. In the left (right) panel, we take $\Delta m_{S}=\Delta m_{H^{ \pm}}=100(200) \mathrm{GeV}$. In the upper (lower) panel, the sign of the $\left|\lambda_{\text {relic }}\right|$ is positive (negative), see eq. (3.22).

\section{B.1 $Z$ boson contribution}

We calculate the contributions from $Z$ boson and its would-be NG boson depicted by the diagrams in figure 11. In the followings, "crossed" means diagrams in which the vertices which $A$ attached are flipped. The box-diagrams without would-be NG bosons (figure 11(b)) contribute to twist-2 operator.

$$
\text { Figure } 11(a)=\frac{i}{(4 \pi)^{2}} g_{Z}^{2} \frac{m_{f}}{m_{Z}^{2}}\left(2 g_{f_{L}} g_{f_{R}}-\frac{1}{4}\left(g_{f_{L}}^{2}+g_{f_{R}}^{2}\right)\right) \text {, }
$$

Figure $11(b)+($ crossed $)=\frac{i}{(4 \pi)^{2}} \frac{1}{2} g_{Z}^{2} m_{f}$

$$
\begin{aligned}
& \times\left(\frac{\left(g_{f_{L}}-g_{f_{R}}\right)^{2}}{2} f_{B 1}+m_{A}^{2}\left(g_{f_{L}}^{2}+g_{f_{R}}^{2}\right)\left(f_{B 2}-3 f_{B 3}\right)+4 m_{A}^{2} g_{f_{L}} g_{f_{R}} f_{B 2}\right) \\
& \left.+\frac{i}{(4 \pi)^{2}} g_{Z}^{2}\left(p^{\mu} q_{\mu} \not p-\frac{1}{4} p^{2} \not\right)\right)\left(g_{f_{L}}^{2}+g_{f_{R}}^{2}\right) 2\left(f_{B 2}-f_{B 3}\right)
\end{aligned}
$$

Figure $11(c), 11(d)+($ crossed $)=-\frac{i}{(4 \pi)^{2}} \frac{1}{2} g_{Z}^{2} \frac{m_{S}^{2}-m_{A}^{2}}{v^{2}} m_{f}\left(f_{B 1}+2 m_{A}^{2} f_{B 4}\right)$, 



Figure 10. The $\sigma_{\mathrm{SI}}$ in $\left(m_{\mathrm{DM}}, m_{H^{ \pm}}\right)$-plain. We take $m_{S}=m_{H^{ \pm}}$. The value of $\sigma_{\mathrm{SI}}$ is $\sigma_{\mathrm{SI}}<$ $10^{-49} \mathrm{~cm}^{2}, 10^{-49} \mathrm{~cm}^{2}<\sigma_{\mathrm{SI}}<10^{-48} \mathrm{~cm}^{2}, 10^{-48} \mathrm{~cm}^{2}<\sigma_{\mathrm{SI}}<10^{-47} \mathrm{~cm}^{2}, 10^{-47} \mathrm{~cm}^{2}<\sigma_{\mathrm{SI}}<$ $10^{-46} \mathrm{~cm}^{2}$, and $10^{-46} \mathrm{~cm}^{2}<\sigma_{\mathrm{SI}}$ in the red, orange, yellow, green, and cyan regions, respectively. From the left to the right panel, we take $\lambda_{2}=0,0.5$, and 1 , respectively. In the upper (lower) panel, the sign of the $\left|\lambda_{\text {relic }}\right|$ is positive (negative), see eq. (3.22).



(a)

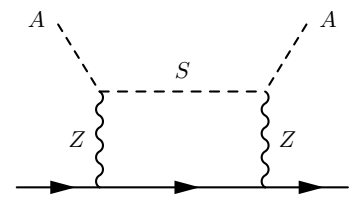

(b)



(c)

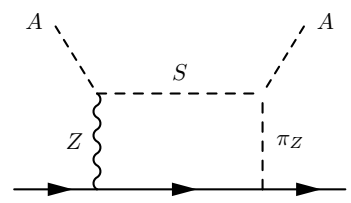

(d)

Figure 11. For the box diagrams, we also have "crossed" diagrams in which the vertices $A$ attached are flipped.

and where $p$ and $q$ are four-momenta of the dark matter and the quark, respectively. Note that we ignore the momentum transfer between the dark matter and the quark. The definitions of $f_{B 1}, f_{B 2}$, and $f_{B 3}$ are given in appendix E.2, and their argument here is $\left(m_{Z}, m_{S}, m_{A}\right)$.

\section{B.2 W boson contribution}

We calculate the contributions from $W$ boson and its would-be NG boson depicted by the diagrams in figure 12. In the followings, "crossed" means diagrams in which the vertices $A$ attached are flipped. The box-diagrams without would-be NG bosons contribute to twist-2 




(a)

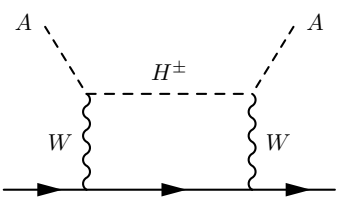

(b)

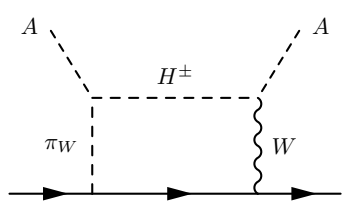

(c)



(d)

Figure 12. For the box diagrams, we also have "crossed" diagrams in which the vertices $A$ attached are flipped.

operator.

$$
\text { Figure } 12(a)=-\frac{1}{8} \frac{i}{(4 \pi)^{2}} g_{W}^{4} \frac{m_{f}}{m_{W}^{2}},
$$

$$
\begin{aligned}
\text { Figure } 12(b)+(\text { crossed })= & \frac{1}{8} \frac{i}{(4 \pi)^{2}} g_{W}^{4} m_{f}\left(f_{B 1}+m_{A}^{2}\left(2 f_{B 2}-6 f_{B 3}\right)\right) \\
& +\frac{i}{(4 \pi)^{2}} g_{W}^{4}\left(p^{\mu} q_{\mu} \not p-\frac{1}{4} p^{2} \not 1\right)\left(f_{B 2}-f_{B 3}\right),
\end{aligned}
$$

Figure $12(c)+$ Figure $12(d)+(\operatorname{crossed})=-\frac{i}{(4 \pi)^{2}} g_{W}^{2} \frac{m_{H^{ \pm}}^{2}-m_{A}^{2}}{v^{2}} m_{f}\left(f_{B 1}+2 m_{A}^{2} f_{B 4}\right)$,

and where $p$ and $q$ are four-momenta of the dark matter and the quark, respectively. Note that we ignore the momentum transfer between the dark matter and the quark. The definitions of $f_{B 1}, f_{B 2}$, and $f_{B 3}$ are given in appendix E.2, and their argument here is $\left(m_{W}, m_{H^{ \pm}}, m_{A}\right)$.

\section{One-loop higgs vertex corrections}

We calculate one-loop corrections to the dark matter coupling to the Higgs boson. We interested in the case that the coupling is highly suppressed at the tree level. Hence we take $\lambda_{A}=0$, in our calculation. We denote $q^{2}$ as the momentum of the Higgs boson, and treat the Higgs boson as off-shell, because what we need is the difference between $q^{2}=m_{h}^{2}$ case and $q^{2}=0$ case. Hence we ignore terms independent from $q^{2}$ in the following calculations. The sum of the diagrams we calculate in this section gives $-i v \delta \Gamma_{h}$, where $\delta \Gamma_{h}$ is defined in eq. (3.1).

\section{C.1 $Z$ boson contribution}

Up to the $q^{2}$-independent terms, we find

Figure $13(a)$

$$
=\frac{2 i}{(4 \pi)^{2}} \frac{m_{Z}^{2}}{v^{2}} \frac{m_{S}^{2}-m_{A}^{2}}{v}\left(F_{1}\left(m_{S}^{2}, q^{2}\right)+\left(-m_{Z}^{2}+2 m_{S}^{2}+2 m_{A}^{2}-2 q^{2}\right) F_{2}\left(m_{S}^{2}, m_{Z}^{2}, q^{2}\right)\right),
$$

Figure $13(b)$

$$
=\frac{2 i}{(4 \pi)^{2}} \frac{m_{Z}^{2}}{v^{2}} \frac{m_{Z}^{2}}{v}\left(-2 F_{1}\left(m_{Z}^{2}, q^{2}\right)+\left(-m_{Z}^{2}+2 m_{S}^{2}+2 m_{A}^{2}-\frac{1}{2} q^{2}\right) F_{2}\left(m_{Z}^{2}, m_{S}^{2}, q^{2}\right)\right),
$$


<smiles>CC1CC(I)CC(I)C1</smiles>

(a)

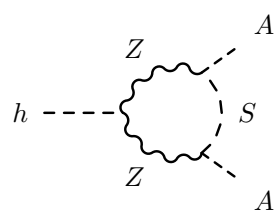

(b)

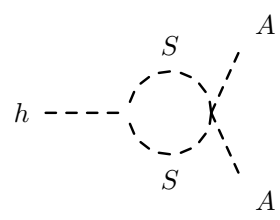

(c)

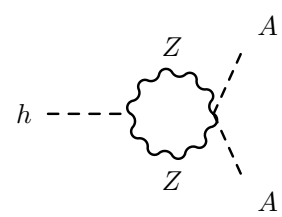

(d)

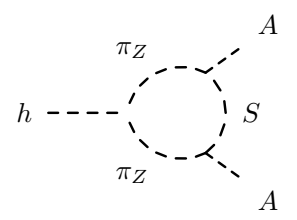

(e)<smiles>CC1CC(I)C(I)CC1I</smiles>

(f)<smiles>CC1CC(I)CC(I)C1</smiles>

(g)



(h)

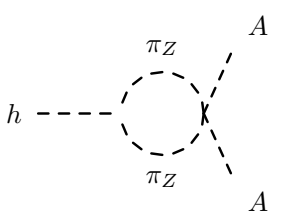

(i)

Figure 13. The diagrams for the vertex correction with neutral particles.

Figure 13(c)

$$
=\frac{2 i}{(4 \pi)^{2}} \lambda_{2} \frac{m_{S}^{2}-m_{A}^{2}}{v} F_{1}\left(m_{S}^{2}, q^{2}\right)
$$

Figure $13(d)$

$$
=\frac{8 i}{(4 \pi)^{2}} \frac{m_{Z}^{2}}{v^{2}} \frac{m_{Z}^{2}}{v} F_{1}\left(m_{Z}^{2}, q^{2}\right)
$$

Figure $13(e)$

$$
=-\frac{i}{(4 \pi)^{2}} \frac{m_{h}^{2}}{v}\left(\frac{m_{S}^{2}-m_{A}^{2}}{v}\right)^{2} F_{2}\left(m_{Z}^{2}, m_{S}^{2}, q^{2}\right),
$$

Figure 13(f)

$$
=-\frac{2 i}{(4 \pi)^{2}}\left(\frac{m_{S}^{2}-m_{A}^{2}}{v}\right)^{3} F_{2}\left(m_{S}^{2}, m_{Z}^{2}, q^{2}\right),
$$

Figure $13(g)+$ Figure $13(h)$

$$
=\frac{2 i}{(4 \pi)^{2}} \frac{m_{Z}^{2}}{v^{2}}\left(\frac{m_{S}^{2}-m_{A}^{2}}{v}\right)\left(F_{1}\left(m_{Z}^{2}, q^{2}\right)-\left(m_{S}^{2}-m_{A}^{2}+q^{2}\right) F_{2}\left(m_{Z}^{2}, m_{S}^{2}, q^{2}\right)\right),
$$

Figure 13(i)

$$
=\frac{i}{(4 \pi)^{2}} \frac{m_{h}^{2}}{v^{2}}\left(\frac{m_{S}^{2}-m_{A}^{2}}{v}\right) F_{1}\left(m_{Z}^{2}, q^{2}\right)
$$

where $F_{1}$ and $F_{2}$ are defined in the appendix E.

\section{C.2 W boson contribution}

Up to the $q^{2}$-independent terms, we find

Figure 14(a) + Figure 14(b)

$$
=\frac{4 i}{(4 \pi)^{2}} \frac{m_{W}^{2}}{v^{2}} \frac{m_{H^{ \pm}}^{2}-m_{A}^{2}}{v}\left(F_{1}\left(m_{H^{ \pm}}^{2}, q^{2}\right)+\left(-m_{W}^{2}+2 m_{H^{ \pm}}^{2}+2 m_{A}^{2}-2 q^{2}\right) F_{2}\left(m_{H^{ \pm}}^{2}, m_{W}^{2}, q^{2}\right)\right),
$$


Figure 14(c) + Figure 14(d)

$$
=-\frac{4 i}{(4 \pi)^{2}} \frac{m_{W}^{4}}{v^{3}}\left(2 F_{1}\left(m_{W}^{2}, q^{2}\right)+\left(m_{W}^{2}-2 m_{H^{ \pm}}^{2}-2 m_{A}^{2}+\frac{1}{2} q^{2}\right) F_{2}\left(m_{W}^{2}, m_{H^{ \pm}}, q^{2}\right)\right),
$$

Figure 14(e)

$$
=\frac{4 i}{(4 \pi)^{2}} \lambda_{2} \frac{m_{H^{ \pm}}^{2}-m_{A}^{2}}{v} F_{1}\left(m_{H^{ \pm}}^{2}, q^{2}\right)
$$

Figure $14(f)$

$$
=\frac{16 i}{(4 \pi)^{2}} \frac{m_{W}^{4}}{v^{3}} F_{1}\left(m_{W}^{2}, q^{2}\right),
$$

Figure 14 $(g)+$ Figure 14 $(h)$

$$
=-\frac{2 i}{(4 \pi)^{2}} \frac{m_{h}^{2}}{v}\left(\frac{m_{H^{ \pm}}^{2}-m_{A}^{2}}{v}\right)^{2} F_{2}\left(m_{W}^{2}, m_{H^{ \pm}}, q^{2}\right),
$$

Figure $14(i)+$ Figure $14(j)$

$$
=-\frac{4 i}{(4 \pi)^{2}}\left(\frac{m_{H^{ \pm}}^{2}-m_{A}^{2}}{v}\right)^{3} F_{2}\left(m_{H^{ \pm}}^{2}, m_{W}^{2}, q^{2}\right),
$$

Figures $14(k)+14(l)+14(m)+14(n)$

$$
=\frac{4 i}{(4 \pi)^{2}} \frac{m_{W}^{2}}{v^{2}} \frac{m_{H^{ \pm}}^{2}-m_{A}^{2}}{v}\left(F_{1}\left(m_{W}^{2}, q^{2}\right)-\left(m_{H^{ \pm}}^{2}-m_{A}^{2}+q^{2}\right) F_{2}\left(m_{W}^{2}, m_{H^{ \pm}}, q^{2}\right)\right),
$$

Figure 14(o)

$$
=\frac{2 i}{(4 \pi)^{2}} \frac{m_{h}^{2}}{v^{2}} \frac{m_{H^{ \pm}}^{2}-m_{A}^{2}}{v} F_{1}\left(m_{W}^{2}, q^{2}\right) .
$$

\section{Gluon contribution at two-loop level}

The effective operator $A_{0}^{2} G_{\mu \nu}^{a} G^{a \mu \nu}$ also give non-negligible contribution. Two-loop diagrams shown in figure 15 give contributions to this operator. The shaded region contains quark loop diagram. There are also would-be Nambu-Goldstone (NG) bosons contributions, but we suppressed them in the figures. The last two diagrams in figure 15 are proportional to $\lambda_{A}$ which is much smaller than the other couplings, so we ignore their contributions. In this subsection, we describe an evaluation of them by taking a method which is used for a calculation of the cross section of wino dark matter-nucleon scattering $[57,61,67]$. Note that the operator with the gluon field strength at two-loop order is the same as the operator without gluon field at one-loop order as we have discussed in section 3.1.

\section{D.1 Two-point functions in the gluon background field}

First, we evaluate quark loop sub-diagrams in the two-loop diagrams shown in figure 16, 17 and 18. For this purpose, we calculate one-loop corrections for two-point functions of gauge boson / pseudo-NG boson in the gluon background field by taking the Fock-Schwinger gauge $x^{\mu} A_{\mu}^{a}=0$ for the gluon field, where $x^{\mu}$ is the position four vector. In the following 
<smiles>CC1CC(C)C(I)C(I)C1</smiles>

(a)



(b)

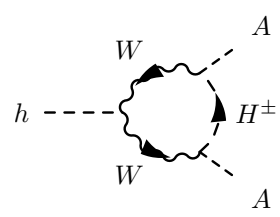

(c)

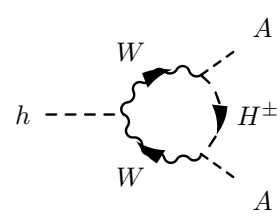

(d)

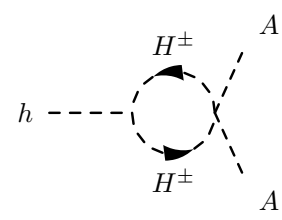

(e)

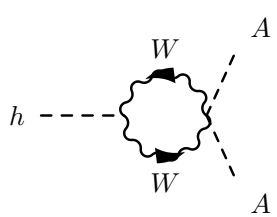

(f)



(g)

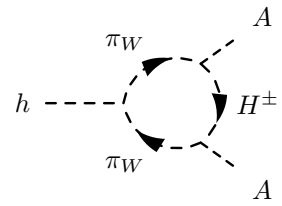

(h)

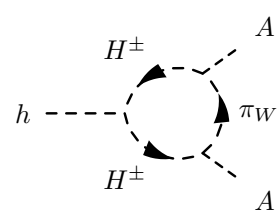

(i)

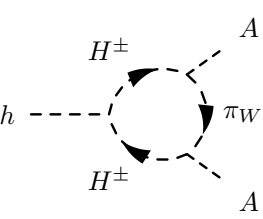

(j)



(k)

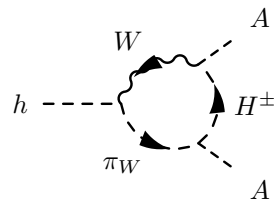

(1)

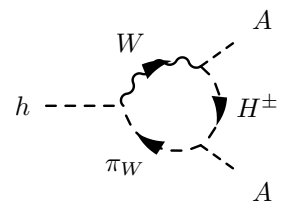

(m)

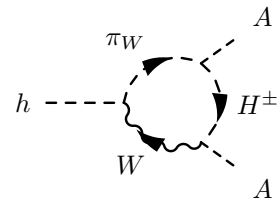

(n)

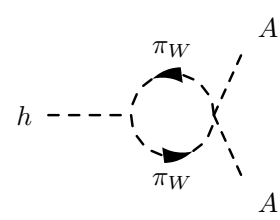

(o)

Figure 14. The diagrams for the vertex correction with charged particles.



(a)



(b)

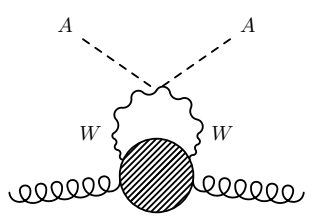

(c)



(d)

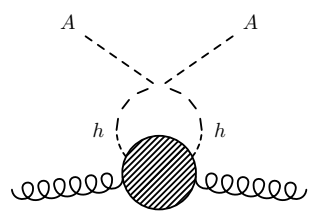

(e)

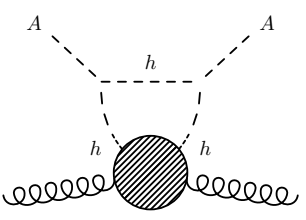

(f)

Figure 15. The diagram we calculate in this section. The shaded quark loop diagram. We suppressed NG boson contributions. The last two diagrams are proportional to $\lambda_{A}$ which is much smaller than the other couplings, so we ignore their contributions.

of this paper, we only take into account gluon twist- 0 operator and neglect higher twist operators, i.e., a product of gluon field strength can be substitute as,

$$
G_{\mu \rho}^{a} G_{\nu \sigma}^{a} \rightarrow \frac{1}{12}\left(g_{\mu \nu} g_{\rho \sigma}-g_{\mu \sigma} g_{\nu \rho}\right) G_{\mu \nu}^{a} G^{a \mu \nu} .
$$

Thanks to these simplifications, two-point function of $W$ boson and pseudo-NG boson $\pi_{W}$ can be factorized as,

$$
i \Pi_{W W}^{(j) \alpha \beta}=-\frac{1}{6} \frac{i g_{s}^{2}}{16 \pi^{2}} G_{\mu \nu}^{a} G^{a \mu \nu}\left(A_{W}^{(j)}\left(q^{2}\right) g^{\alpha \beta}+B_{W}^{(j)}\left(q^{2}\right) q^{\alpha} q^{\beta}\right),
$$




$$
\begin{aligned}
i \Pi_{W \pi_{W}}^{(j) \alpha} & =-\frac{1}{6} \frac{i g_{s}^{2}}{16 \pi^{2}} G_{\mu \nu}^{a} G^{a \mu \nu} C_{W}^{(j)}\left(q^{2}\right) q^{\alpha}, \\
i \Pi_{\pi_{W} \pi_{W}}^{(j)} & =-\frac{1}{6} \frac{i g_{s}^{2}}{16 \pi^{2}} G_{\mu \nu}^{a} G^{a \mu \nu} D_{W}^{(j)}\left(q^{2}\right),
\end{aligned}
$$

where $j=1,2$ and 3 express generation of quarks which give the contribution to the two-point function. Also, for $Z$ boson and $\pi_{Z}$,

$$
\begin{aligned}
i \Pi_{Z Z}^{(f) \alpha \beta} & =-\frac{1}{6} \frac{i g_{s}^{2}}{16 \pi^{2}} G_{\mu \nu}^{a} G^{a \mu \nu}\left(A_{Z}^{(f)}\left(q^{2}\right) g^{\alpha \beta}+B_{Z}^{(f)}\left(q^{2}\right) q^{\alpha} q^{\beta}\right), \\
i \Pi_{Z \pi_{Z}}^{(f) \alpha} & =-\frac{1}{6} \frac{i g_{s}^{2}}{16 \pi^{2}} G_{\mu \nu}^{a} G^{a \mu \nu} C_{Z}^{(f)}\left(q^{2}\right) q^{\alpha} \\
i \Pi_{\pi_{Z} \pi_{Z}}^{(f)} & =-\frac{1}{6} \frac{i g_{s}^{2}}{16 \pi^{2}} G_{\mu \nu}^{a} G^{a \mu \nu} D_{Z}^{(f)}\left(q^{2}\right),
\end{aligned}
$$

where $f=u, d, s, c, b$ and $t$. In $\Pi_{W \pi_{W}}^{(i) \alpha}\left(q^{2}\right)$ and $\Pi_{Z \pi_{Z}}^{(f) \alpha}\left(q^{2}\right), q$ is momentum of gauge boson and its direction is out-going.

As noted in refs. [57, 61, 67], for the evaluation of the above two-point functions, we have to be careful for double-counting. The loop integral in diagram 16(b), 16(c), 17(b), 17(c), 18(b) and 18(c) dominates when the internal momentum is around a mass of quark emitting gluons. In these diagrams, if the quark emitting gluons is light quarks (i.e., up, down or strange), the dominant contribution comes from a region in which the internal momentum is smaller than QCD confinement scale. In such a region, perturbative calculation cannot be reliable, and the corresponding effect should be included in the evaluation of $\left\langle N\left|m_{q} \bar{q} q\right| N\right\rangle$ [68]. Therefore, the diagrams in which up, down or strange quark emitting two gluons should be removed in the evaluation of the above $A, B, C$ and $D$ function. On the other hand, the loop integral in diagram 16(a), 17(a) and 18(a) dominates when the internal momentum is around external momentum $q$, which is the order of $m_{W}$ or $m_{Z}$. Therefore, this diagram always should be took into account of all of the quarks. We assume $m_{c}$ and $m_{b}$ is larger than QCD confinement scale, but much smaller than $m_{W}, m_{t}$, $m_{A}$. The charged gauge/pseudo-NG bosons obtain the contributions from up and down quark as,

$$
A_{W}^{(1)}\left(q^{2}\right)=\frac{g_{W}^{2}}{2} \frac{1}{q^{2}}, \quad B_{W}^{(1)}\left(q^{2}\right)=-\frac{g_{W}^{2}}{2} \frac{1}{q^{4}}, \quad C_{W}^{(1)}\left(q^{2}\right)=0, \quad D_{W}^{(1)}\left(q^{2}\right)=0 .
$$

From charm and strange quark,

$$
A_{W}^{(2)}\left(q^{2}\right)=\frac{g_{W}^{2}}{2} \frac{1}{q^{2}}, \quad B_{W}^{(2)}\left(q^{2}\right)=0, \quad C_{W}^{(2)}\left(q^{2}\right)=\frac{g_{W}^{2}}{2} \frac{1}{2 m_{W}} \frac{2}{q^{2}}, \quad D_{W}^{(2)}\left(q^{2}\right)=0 .
$$

From top and bottom quark,

$$
\begin{aligned}
A_{W}^{(3)} & =\frac{g_{W}^{2}}{2}\left(\frac{1}{q^{2}-m_{t}^{2}}-\frac{1}{2} \frac{m_{t}^{2}}{\left(q^{2}-m_{t}^{2}\right)^{2}}\right), & B_{W}^{(3)}\left(q^{2}\right) & =\frac{g_{W}^{2}}{2} \frac{1}{\left(q^{2}-m_{t}^{2}\right)^{2}}, \\
C_{W}^{(3)}\left(q^{2}\right) & =\frac{g_{W}^{2}}{2} \frac{1}{2 m_{W}} \frac{4 q^{2}-3 m_{t}^{2}}{\left(q^{2}-m_{t}^{2}\right)^{2}}, & D_{W}^{(3)}\left(q^{2}\right) & =\frac{g_{W}^{2}}{2} \frac{m_{t}^{2}}{2 m_{W}^{2}} \frac{5 q^{2}-4 m_{t}^{2}}{\left(q^{2}-m_{t}^{2}\right)^{2}} .
\end{aligned}
$$




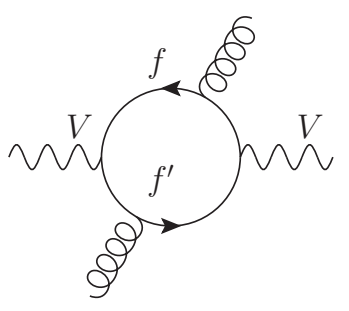

(a)



(b)



(c)

Figure 16. One-loop corrections for two point function of gauge boson in gluon background field.

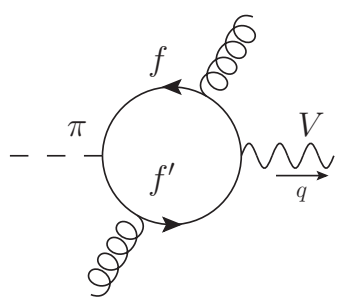

(a)

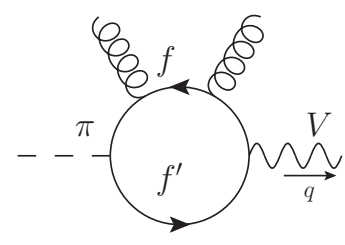

(b)



(c)

Figure 17. One-loop corrections for two point function of gauge boson and pseudo-NG boson in gluon background field.



(a)

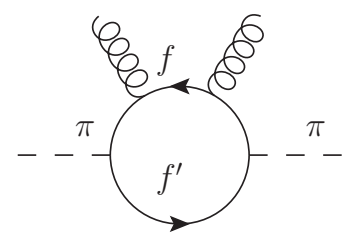

(b)

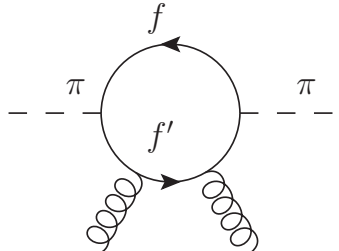

(c)

Figure 18. One-loop corrections for two point function of pseudo-NG boson in gluon background field.

Neutral current couplings of quark $f$ are defined as $g_{f_{L}}=g_{Z}\left(T_{3 f}-s_{W}^{2} Q_{f}\right)$ and $g_{f_{R}}=$ $-g_{Z} s_{W}^{2} Q_{f}$. The neutral gauge/pseudo-NG bosons obtain the contributions from up, down and strange quark as,

$$
A_{Z}^{(f)}=\frac{g_{f_{L}}^{2}+g_{f_{R}}^{2}}{q^{2}}, \quad B_{Z}^{(f)}\left(q^{2}\right)=-\frac{g_{f_{L}}^{2}+g_{f_{R}}^{2}}{q^{4}}, \quad C_{Z}^{(f)}\left(q^{2}\right)=0, \quad D_{Z}^{(f)}\left(q^{2}\right)=0 .
$$

From charm and bottom quark,

$$
A_{Z}^{(f)}=\frac{g_{f_{L}}^{2}-4 g_{f_{L}} g_{f_{R}}+g_{f_{R}}^{2}}{q^{2}}, \quad B_{Z}^{(f)}\left(q^{2}\right)=\frac{g_{f_{L}}^{2}+g_{f_{R}}^{2}}{q^{4}}, \quad C_{Z}^{(f)}\left(q^{2}\right)=\frac{g_{Z}^{2}}{2 m_{Z}} \frac{1}{q^{2}}, \quad D_{Z}^{(f)}\left(q^{2}\right)=0 .
$$


From top quark,

$$
\begin{aligned}
A_{Z}^{(t)}\left(q^{2}\right)= & \left(g_{t_{L}}^{2}+g_{t_{R}}^{2}\right) \int_{0}^{1} d x\left(\frac{-w(1-w)}{\Delta(w)}+\frac{m_{t}^{2}\left(2-5 w+5 w^{2}\right)}{[\Delta(w)]^{2}}+\frac{m_{t}^{4}\left(-2+6 w-6 w^{2}\right)}{[\Delta(w)]^{3}}\right) \\
& +\frac{g_{Z}^{2}}{4} \int_{0}^{1} d x\left(\frac{m_{t}^{2}\left(-1+2 w-2 w^{2}\right)}{[\Delta(w)]^{2}}+\frac{m_{t}^{4} w(1-w)}{[\Delta(w)]^{3}}\right), \\
B_{Z}^{(t)}\left(q^{2}\right)= & -\left(g_{t_{L}}^{2}+g_{t_{R}}^{2}\right) \int_{0}^{1} d w\left[\frac{w^{2}(1-w)^{2}}{[\Delta(w)]^{2}}-\frac{2 m_{t}^{2} w(1-w)\left(1-3 w+3 w^{2}\right)}{[\Delta(w)]^{3}}\right], \\
C_{Z}^{(t)}\left(q^{2}\right)= & -\frac{3 g_{Z}^{2}}{4} \frac{m_{t}^{2}}{m_{Z}} \int_{0}^{1} d w\left[\frac{1-2 w+2 w^{2}}{2[\Delta(w)]^{2}}-\frac{m_{t}^{2}\left(1-3 w+3 w^{2}\right)}{3[\Delta(w)]^{3}}\right], \\
D_{Z}^{(t)}\left(q^{2}\right)= & \frac{g_{Z}^{2}}{4} \frac{m_{t}^{2}}{m_{Z}^{2}} \int_{0}^{1} d w\left[-\frac{3 w(1-w)}{\Delta(w)}-\frac{3 m_{t}^{2}\left(1+w-w^{2}\right)}{[\Delta(w)]^{2}}+\frac{4 m_{t}^{4}\left(1-3 w+3 w^{2}\right)}{[\Delta(w)]^{3}}\right],
\end{aligned}
$$

where $\Delta(w) \equiv m_{t}^{2}-w(1-w) q^{2}$.

\section{D.2 Effective interaction for dark matter-gluon scattering}

Next, by using the self-energy functions which have been evaluated so far, we evaluate the $\Gamma_{\mathrm{Box}}^{G}$ which is the coefficient of the effective operator $A^{2} G_{\mu \nu}^{a} G^{a \mu \nu}$ as defined in eq. (3.1). We take the Feynman-'t Hooft gauge for electroweak gauge bosons, and find $\Gamma_{\text {Box }}^{G}$ is expressed as,

$$
\begin{aligned}
&-\frac{\alpha_{s}}{4 \pi} \Gamma_{\text {Box }}^{G}= \sum_{i} f_{G, W}^{(i)}+\sum_{f} f_{G, Z}^{(f)}, \\
& f_{G, W}^{(i)}= \frac{i g_{W}^{2}}{12} \frac{g_{s}^{2}}{16 \pi^{2}} \sum_{i} \int \frac{d^{d} \ell}{(2 \pi)^{d}}\left[\frac{3 \ell^{2}+4 \ell p-4 m_{H}^{2}}{\left[(\ell+p)^{2}-m_{H}^{2}\right]\left[\ell^{2}-m_{W}^{2}\right]^{2}} A_{W}^{(i)}\left(\ell^{2}\right)\right. \\
&\left.-\frac{m_{H}^{2}-m_{A}^{2}}{m_{W}^{2}} \frac{\ell^{2}+2 \ell p}{\left[(\ell+p)^{2}-m_{H}^{2}\right]\left[\ell^{2}-m_{W}^{2}\right]^{2}} \tilde{B}_{W}^{(i)}\left(\ell^{2}\right)\right], \\
& f_{G, Z}^{(f)}= \frac{i g_{Z}^{2}}{24} \frac{g_{s}^{2}}{16 \pi^{2}} \sum_{f} \int \frac{d^{d} \ell}{(2 \pi)^{d}}\left[\frac{3 \ell^{2}+4 \ell p-4 m_{S}^{2}}{\left[(\ell+p)^{2}-m_{S}^{2}\right]\left[\ell^{2}-m_{Z}^{2}\right]^{2}} A_{Z}^{(f)}\left(\ell^{2}\right)\right. \\
&\left.-\frac{m_{S}^{2}-m_{A}^{2}}{m_{Z}^{2}} \frac{\ell^{2}+2 \ell p}{\left[(\ell+p)^{2}-m_{S}^{2}\right]\left[\ell^{2}-m_{Z}^{2}\right]^{2}} \tilde{B}_{Z}^{(f)}\left(\ell^{2}\right)\right],
\end{aligned}
$$

where $\tilde{B}_{W}^{(i)} \equiv m_{W}^{2} B_{W}^{(i)}-2 m_{W} C_{W}^{(i)}+D_{W}^{(i)}$ and $\tilde{B}_{Z}^{(f)} \equiv m_{Z}^{2} B_{Z}^{(f)}-2 m_{Z} C_{Z}^{(f)}+D_{Z}^{(f)} \cdot X_{n}$ and $Y_{n}$ which are defined in the appendix E.3 are useful for the evaluations of two-loop diagrams. For the convenience, we define $X_{n}^{W q}, X_{n}^{W t}, X_{n}^{Z q}$ and $\tilde{X}_{n}^{Z t}$ as,

$$
\begin{aligned}
X_{n}^{W q} & \equiv X_{n}\left(m_{A}^{2}, m_{H^{ \pm}}^{2}, m_{W}^{2}, 0\right), \\
X_{n}^{W t} & \equiv X_{n}\left(m_{A}^{2}, m_{H^{ \pm}}^{2}, m_{W}^{2}, m_{t}^{2}\right), \\
X_{n}^{Z q} & \equiv X_{n}\left(m_{A}^{2}, m_{S}^{2}, m_{Z}^{2}, 0\right), \\
\tilde{X}_{n}^{Z t}(w) & \equiv X_{n}\left(m_{A}^{2}, m_{S}^{2}, m_{Z}^{2}, w^{-1}(1-w)^{-1} m_{t}^{2}\right) .
\end{aligned}
$$


$Y_{n}^{W q}, Y_{n}^{W t}, Y_{n}^{Z q}$ and $\tilde{Y}_{n}^{Z t}(w)$ are also defined in the same manner. Finally, the contributions to $f_{G}$ is written as,

$$
\begin{aligned}
& f_{G, W}^{(1)}=-\frac{g_{W}^{2} g_{s}^{2}}{12\left(16 \pi^{2}\right)^{2}} \frac{g_{W}^{2}}{2}\left(3 X_{0}^{W q}+4 m_{A}^{2} Y_{1}^{W q}-4 m_{H^{ \pm}}^{2} X_{1}^{W q}+\left(m_{H^{ \pm}}^{2}-m_{A}^{2}\right)\left(X_{1}^{W q}+2 m_{A}^{2} Y_{2}^{W q}\right)\right), \\
& f_{G, W}^{(2)}=-\frac{g_{W}^{2} g_{s}^{2}}{12\left(16 \pi^{2}\right)^{2}} \frac{g_{W}^{2}}{2}\left(3 X_{0}^{W q}+4 m_{A}^{2} Y_{1}^{W q}-4 m_{H^{ \pm}}^{2} X_{1}^{W q}\right. \\
& \left.+\frac{2}{m_{W}^{2}}\left(m_{H^{ \pm}}^{2}-m_{A}^{2}\right)\left(X_{0}^{W q}+2 m_{A}^{2} Y_{1}^{W q}\right)\right), \\
& f_{G, W}^{(3)}=-\frac{g_{W}^{2} g_{s}^{2}}{12\left(16 \pi^{2}\right)^{2}} \frac{g_{W}^{2}}{2}\left[\left(3 X_{0}^{W t}+4 m_{A}^{2} Y_{1}^{W t}+\left(3 m_{t}^{2}-4 m_{H^{ \pm}}^{2}\right) X_{1}^{W t}\right)\right. \\
& -\frac{m_{t}^{2}}{2}\left(3 X_{1}^{W t}+4 m_{A}^{2} Y_{2}^{W t}+\left(3 m_{t}^{2}-4 m_{H^{ \pm}}^{2}\right) X_{2}^{W t}\right) \\
& -\frac{m_{H^{ \pm}}^{2}-m_{A}^{2}}{m_{W}^{2}}\left(-4+\frac{5 m_{t}^{2}}{2 m_{W}^{2}}\right)\left(X_{0}^{W t}+2 m_{A}^{2} Y_{1}^{W t}+m_{t}^{2} X_{1}^{W t}\right) \\
& \left.-\left(m_{H^{ \pm}}^{2}-m_{A}^{2}\right)\left(1-\frac{m_{t}^{2}}{m_{W}^{2}}+\frac{m_{t}^{4}}{2 m_{W}^{4}}\right)\left(X_{1}^{W t}+2 m_{A}^{2} Y_{2}^{W t}+m_{t}^{2} X_{2}^{W t}\right)\right] .
\end{aligned}
$$

For up, down and strange quarks $(f=u, d, s)$,

$f_{G, Z}^{(f)}=-\frac{g_{Z}^{2} g_{s}^{2}}{24\left(16 \pi^{2}\right)^{2}}\left(g_{f_{L}}^{2}+g_{f_{R}}^{2}\right)\left[\left(3 X_{0}^{Z q}+4 m_{A}^{2} Y_{1}^{Z q}-4 m_{S}^{2} X_{1}^{Z q}\right)+\left(m_{S}^{2}-m_{A}^{2}\right)\left(X_{1}^{Z q}+2 m_{A}^{2} Y_{2}^{Z q}\right)\right]$.

For charm and bottom quarks $(f=c, b)$,

$$
\begin{aligned}
f_{G, Z}^{(f)}=-\frac{g_{Z}^{2} g_{s}^{2}}{24\left(16 \pi^{2}\right)^{2}} & {\left[\left(g_{f_{L}}^{2}-4 g_{f_{L}} g_{f_{R}}+g_{f_{R}}^{2}\right)\left(3 X_{0}^{Z q}+4 m_{A}^{2} Y_{1}^{Z q}-4 m_{S}^{2} X_{1}^{Z q}\right)\right.} \\
& \left.-\frac{m_{S}^{2}-m_{A}^{2}}{m_{Z}^{2}}\left[-g_{Z}^{2}\left(X_{0}^{Z q}+2 m_{A}^{2} Y_{1}^{Z q}\right)+\left(g_{f_{L}}^{2}+g_{f_{R}}^{2}\right) m_{Z}^{2}\left(X_{1}^{Z q}+2 m_{A}^{2} Y_{2}^{Z q}\right)\right]\right] .
\end{aligned}
$$

For top-quark,

$$
\begin{aligned}
f_{G, Z}^{(t)}= & -\frac{g_{Z}^{2} g_{s}^{2}}{24\left(16 \pi^{2}\right)^{2}} \sum_{n=1}^{3} \int_{0}^{1} d w \frac{(-1)^{n} m_{t}^{2(n-1)}}{w^{n}(1-w)^{n}} \\
\times & {\left[\left(3 \tilde{X}_{n-1}^{Z t}(w)+4 m_{A}^{2} \tilde{Y}_{n}^{Z t}(w)+\left(\frac{3 m_{t}^{2}}{w(1-w)}-4 m_{S}^{2}\right) \tilde{X}_{n}^{Z t}(w)\right) g_{A n}(w)\right.} \\
& \left.\quad-\frac{m_{S}^{2}-m_{A}^{2}}{m_{Z}^{2}}\left(\tilde{X}_{n-1}^{Z t}(w)+2 m_{A}^{2} \tilde{Y}_{n}^{Z t}(w)+\frac{m_{t}^{2}}{w(1-w)} \tilde{X}_{n}^{Z t}(w)\right) g_{B n}(w)\right] .
\end{aligned}
$$

Here, $g_{A n}$ and $g_{B n}$ are functions which satisfy,

$$
\begin{aligned}
& A_{Z}^{(t)}\left(q^{2}\right)=\int_{0}^{1} d w\left(\frac{g_{A 1}(w)}{\Delta(w)}+\frac{m_{t}^{2} g_{A 2}(w)}{[\Delta(w)]^{2}}+\frac{m_{t}^{4} g_{A 3}(w)}{[\Delta(w)]^{3}}\right), \\
& \tilde{B}_{Z}^{(t)}\left(q^{2}\right)=\int_{0}^{1} d w\left(\frac{g_{B 1}(w)}{\Delta(w)}+\frac{m_{t}^{2} g_{B 2}(w)}{[\Delta(w)]^{2}}+\frac{m_{t}^{4} g_{B 3}(w)}{[\Delta(w)]^{3}}\right),
\end{aligned}
$$


where $\Delta(w)=m_{t}^{2}-w(1-w) q^{2}$. Explicit form of $g_{A n}$ and $g_{B n}$ are given by,

$$
\begin{aligned}
g_{A 1}(w)= & -\left(g_{t_{L}}^{2}+g_{t_{R}}^{2}\right) w(1-w), \\
g_{A 2}(w)= & \left(g_{t_{L}}^{2}+g_{t_{R}}^{2}\right)\left(2-5 w+5 w^{2}\right)+\frac{g_{Z}^{2}}{4}\left(-1+2 w-2 w^{2}\right), \\
g_{A 3}(w)= & \left(g_{t_{L}}^{2}+g_{t_{R}}^{2}\right)\left(-2+6 w-6 w^{2}\right)+\frac{g_{Z}^{2}}{4} w(1-w), \\
g_{B 1}(w)= & -\frac{3 g_{Z}^{2}}{4} \frac{m_{t}^{2}}{m_{Z}^{2}} w(1-w), \\
g_{B 2}(w)= & -\left(g_{t_{L}}^{2}+g_{t_{R}}^{2}\right) \frac{m_{Z}^{2}}{m_{t}^{2}} w^{2}(1-w)^{2}+\frac{g_{Z}^{2}}{4}\left(3-6 w+6 w^{2}\right)+\frac{g_{Z}^{2}}{4} \frac{m_{t}^{2}}{m_{Z}^{2}}\left(-3-3 w+3 w^{2}\right), \\
g_{B 3}(w)= & \left(g_{t_{L}}^{2}+g_{t_{R}}^{2}\right) \frac{m_{Z}^{2}}{m_{t}^{2}} w(1-w)\left(2-6 w+6 w^{2}\right)+\frac{g_{Z}^{2}}{4}\left(-2+6 w-6 w^{2}\right) \\
& +\frac{g_{Z}^{2}}{4} \frac{m_{t}^{2}}{m_{Z}^{2}}\left(4-12 w+12 w^{2}\right) .
\end{aligned}
$$

\section{E Loop functions for radiative corrections}

In this appendix, we summarize loop functions which are useful for the evaluation of the radiative correction on the spin-independent cross section. $B_{i}, B_{i}^{\prime}, C_{i}$ and $D_{i}$ functions which appears in this appendix are the Passarino-Veltman functions [69] and the derivative with respect to the momentum. Our convention is same as used by LoopTools [70]. The explicit definitions of Passarino-Veltman functions are given as,

$$
\begin{aligned}
& \int \frac{d^{4} \ell}{(2 \pi)^{d}} \frac{1}{\left[\ell^{2}-m_{Z}^{2}\right]\left[(\ell+p)^{2}-m_{S}^{2}\right]}=\frac{i}{16 \pi^{2}} B_{0}\left(p^{2}, m_{Z}^{2}, m_{S}^{2}\right), \\
& \int \frac{d^{4} \ell}{(2 \pi)^{d}} \frac{\ell^{\mu}}{\left[\ell^{2}-m_{Z}^{2}\right]\left[(\ell+p)^{2}-m_{S}^{2}\right]}=\frac{i}{16 \pi^{2}} p^{\mu} B_{1}\left(p^{2}, m_{Z}^{2}, m_{S}^{2}\right), \\
& \int \frac{d^{4} \ell}{(2 \pi)^{d}} \frac{1}{\left[\ell^{2}-m_{Z}^{2}\right]^{2}\left[(\ell+p)^{2}-m_{S}^{2}\right]}=\frac{i}{16 \pi^{2}} C_{0}\left(0, p^{2}, p^{2}, m_{Z}^{2}, m_{Z}^{2}, m_{S}^{2}\right), \\
& \int \frac{d^{4} \ell}{(2 \pi)^{d}} \frac{\ell^{\mu}}{\left[\ell^{2}-m_{Z}^{2}\right]^{2}\left[(\ell+p)^{2}-m_{S}^{2}\right]}=\frac{i}{16 \pi^{2}} p^{\mu} C_{2}\left(0, p^{2}, p^{2}, m_{Z}^{2}, m_{Z}^{2}, m_{S}^{2}\right), \\
& \int \frac{d^{d} \ell}{(2 \pi)^{d}} \frac{i}{\left[\ell^{2}-m_{Z}^{2}\right]^{3}\left[(\ell+p)^{2}-m_{S}^{2}\right]}=\frac{i}{16 \pi^{2}} D_{0}\left(0,0, p^{2}, p^{2}, 0, p^{2}, m_{Z}^{2}, m_{Z}^{2}, m_{Z}^{2}, m_{S}^{2}\right), \\
& \int \frac{d^{d} \ell}{(2 \pi)^{d}} \frac{i}{\left[\ell^{2}-m_{Z}^{3}\right]^{3}\left[(\ell+p)^{2}-m_{S}^{2}\right]}=\frac{\ell^{\mu}}{16 \pi^{2}} p^{\mu} D_{3}\left(0,0, p^{2}, p^{2}, 0, p^{2}, m_{Z}^{2}, m_{Z}^{2}, m_{Z}^{2}, m_{S}^{2}\right) .
\end{aligned}
$$

\section{E.1 One-loop vertex}

The functions $F_{1}$ and $F_{2}$ which are used in the appendix $\mathrm{C}$ are defined as,

$$
\begin{aligned}
F_{1}\left(m^{2}, q^{2}\right) & =B_{0}\left(q^{2}, m^{2}, m^{2}\right), \\
F_{2}\left(m_{1}^{2}, m_{2}^{2}, q^{2}\right) & =-C_{0}\left(q^{2}, m_{A}^{2}, m_{A}^{2}, m_{1}^{2}, m_{1}^{2}, m_{2}^{2}\right) .
\end{aligned}
$$




\section{E.2 One-loop box diagrams}

The functions $f_{B 1}, f_{B 2}, f_{B 3}$ and $f_{B 4}$ which are used in the appendix $\mathrm{B}$ are defined as,

$$
\begin{aligned}
& f_{B 1}\left(m_{1}, m_{2}, m_{A}\right) \equiv \int_{0}^{1} d x \frac{x}{m_{1}^{2} x+m_{2}^{2}(1-x)-m_{A}^{2} x(1-x)} \\
&=-\frac{\partial}{\partial m_{1}^{2}} B_{0}\left(m_{A}^{2}, m_{1}^{2}, m_{2}^{2}\right) \\
& f_{B 2}\left(m_{1}, m_{2}, m_{A}\right) \equiv \int_{x y z} \frac{y(1-z)}{\left(m_{1}^{2} y+m_{2}^{2} z-m_{A}^{2} z(1-z)\right)^{2}} \\
&= \frac{1}{m_{1}^{2}} \frac{\partial}{\partial m_{1}^{2}} B_{0}\left(m_{A}^{2}, m_{1}^{2}, m_{2}^{2}\right)+\frac{1}{m_{1}^{2}} B_{0}^{\prime}\left(m_{A}^{2}, m_{1}^{2}, m_{2}^{2}\right) \\
&+\frac{1}{m_{1}^{4}}\left(B_{1}\left(m_{A}^{2}, m_{2}^{2}, m_{1}^{2}\right)-B_{1}\left(m_{A}^{2}, m_{2}^{2}, 0\right)\right) \\
& f_{B 3}\left(m_{1}, m_{2}, m_{A}\right) \equiv \int_{x y z} \frac{y^{2}}{\left(m_{1}^{2} y+m_{2}^{2} z-m_{A}^{2} z(1-z)\right)^{2}} \\
&= \frac{1}{m_{1}^{2}} \frac{\partial}{\partial m_{1}^{2}} B_{0}\left(m_{A}^{2}, m_{1}^{2}, m_{2}^{2}\right)+\frac{1}{m_{1}^{2}} B_{0}^{\prime}\left(m_{A}^{2}, m_{1}^{2}, m_{2}^{2}\right) \\
&+\frac{1}{m_{1}^{4}}-2 \frac{m_{2}^{2}-m_{A}^{2}}{m_{1}^{6}}\left(B_{1}\left(m_{A}^{2}, m_{1}^{2}, m_{2}^{2}\right)-B_{1}\left(m_{A}^{2}, 0, m_{2}^{2}\right)\right) \\
&+2 \frac{m_{A}^{2}}{m_{1}^{6}}\left(B_{11}\left(m_{A}^{2}, m_{1}^{2}, m_{2}^{2}\right)-B_{11}\left(m_{A}^{2}, 0, m_{2}^{2}\right)\right) \\
& \equiv \int_{x y z} \frac{1}{\left(m_{1}^{2} y+m_{2}^{2} z-m_{A}^{2} z(1-z)\right)^{2}} \\
&=-\frac{1}{m_{1}^{2}} B_{0}^{\prime}\left(m_{A}^{2}, m_{1}^{2}, m_{2}^{2}\right)+\frac{1}{m_{1}^{4}}\left(B_{1}\left(m_{A}^{2}, m_{1}^{2}, m_{2}^{2}\right)-B_{1}\left(m_{A}^{2}, 0, m_{2}^{2}\right)\right) . \\
& f_{B 4}\left(m_{1}, m_{2}, m_{A} .\right.
\end{aligned}
$$

Here, $\int_{x y z}$ is defined as,

$$
\int_{x y z} f(x, y, z) \equiv \int_{x+y+z=1} f(x, y, z) \equiv \int_{0}^{1} d z \int_{0}^{1-z} d y f(1-y-z, y, z) .
$$

\section{E.3 Loop functions for dark matter-gluon scattering}

Here, we summarize some loop functions which are useful for the evaluation of the coefficient of effective interaction between dark matter and gluon.

\section{E.3.1 Definitions of $X, Y$ functions}

We define the following two types of loop functions:

$$
\begin{aligned}
& \int \frac{d^{4} \ell}{(2 \pi)^{4}} \frac{1}{\left[(\ell+p)^{2}-m_{S}^{2}\right]\left[\ell^{2}-m_{Z}^{2}\right]^{2}\left[\ell^{2}-m_{t}^{2}\right]^{n}}=\frac{i}{16 \pi^{2}} X_{n}\left(p^{2}, m_{S}^{2}, m_{Z}^{2}, m_{t}^{2}\right) \\
& \int \frac{d^{4} \ell}{(2 \pi)^{4}} \frac{\ell^{\mu}}{\left[(\ell+p)^{2}-m_{S}^{2}\right]\left[\ell^{2}-m_{Z}^{2}\right]^{2}\left[\ell^{2}-m_{t}^{2}\right]^{n}}=\frac{i}{16 \pi^{2}} p^{\mu} Y_{n}\left(p^{2}, m_{S}^{2}, m_{Z}^{2}, m_{t}^{2}\right) .
\end{aligned}
$$




\section{E.3.2 $X, Y$ in $B, C, D$-function}

$X$ and $Y$ functions which are defined in the previous subsection are rewritten by PassarinoVeltman functions [69]:

$$
\begin{aligned}
& X_{0}\left(m_{A}^{2}, m_{S}^{2}, m_{Z}^{2}, m_{t}^{2}\right)=C_{0}^{(Z)}, \\
& X_{1}\left(m_{A}^{2}, m_{S}^{2}, m_{Z}^{2}, m_{t}^{2}\right)=-\frac{C_{0}^{(Z)}}{m_{t}^{2}-m_{Z}^{2}}+\frac{B_{0}^{(t)}-B_{0}^{(Z)}}{\left(m_{t}^{2}-m_{Z}^{2}\right)^{2}}, \\
& X_{2}\left(m_{A}^{2}, m_{S}^{2}, m_{Z}^{2}, m_{t}^{2}\right)=\frac{C_{0}^{(t)}+C_{0}^{(Z)}}{\left(m_{t}^{2}-m_{Z}^{2}\right)^{2}}+\frac{-2 B_{0}^{(t)}+2 B_{0}^{(Z)}}{\left(m_{t}^{2}-m_{Z}^{2}\right)^{3}}, \\
& X_{3}\left(m_{A}^{2}, m_{S}^{2}, m_{Z}^{2}, m_{t}^{2}\right)=\frac{D_{0}^{(t)}}{\left(m_{t}^{2}-m_{Z}^{2}\right)^{2}}+\frac{-2 C_{0}^{(t)}-C_{0}^{(Z)}}{\left(m_{t}^{2}-m_{Z}^{2}\right)^{3}}+\frac{3 B_{0}^{(t)}-3 B_{0}^{(Z)}}{\left(m_{t}^{2}-m_{Z}^{2}\right)^{4}}, \\
& Y_{1}\left(m_{A}^{2}, m_{S}^{2}, m_{Z}^{2}, m_{t}^{2}\right)=-\frac{C_{2}^{(Z)}}{m_{t}^{2}-m_{Z}^{2}}+\frac{B_{1}^{(t)}-B_{1}^{(Z)}}{\left(m_{t}^{2}-m_{Z}^{2}\right)^{2}}, \\
& Y_{2}\left(m_{A}^{2}, m_{S}^{2}, m_{Z}^{2}, m_{t}^{2}\right)=\frac{C_{2}^{(t)}+C_{2}^{(Z)}}{\left(m_{t}^{2}-m_{Z}^{2}\right)^{2}}+\frac{-2 B_{1}^{(t)}+2 B_{1}^{(Z)}}{\left(m_{t}^{2}-m_{Z}^{2}\right)^{3}}, \\
& Y_{3}\left(m_{A}^{2}, m_{S}^{2}, m_{Z}^{2}, m_{t}^{2}\right)=\frac{D_{3}^{(t)}}{\left(m_{t}^{2}-m_{Z}^{2}\right)^{2}}+\frac{-2 C_{2}^{(t)}-C_{2}^{(Z)}}{\left(m_{t}^{2}-m_{Z}^{2}\right)^{3}}+\frac{3 B_{1}^{(t)}-3 B_{1}^{(Z)}}{\left(m_{t}^{2}-m_{Z}^{2}\right)^{4}},
\end{aligned}
$$

where $B_{i}^{(X)}, C_{i}^{(X)}$ and $D_{i}^{(X)}$ are

$$
\begin{aligned}
B_{i}^{(X)} & \equiv B_{i}\left(m_{A}^{2}, m_{X}^{2}, m_{S}^{2}\right) \\
C_{i}^{(X)} & \equiv C_{i}\left(0, m_{A}^{2}, m_{A}^{2}, m_{X}^{2}, m_{X}^{2}, m_{S}^{2}\right) \\
D_{i}^{(X)} & \equiv D_{i}\left(0,0, m_{A}^{2}, m_{A}^{2}, 0, m_{A}^{2}, m_{X}^{2}, m_{X}^{2}, m_{X}^{2}, m_{S}^{2}\right) .
\end{aligned}
$$

\section{E.3.3 $C, D$ in $B_{0}$ and $\partial B_{0} / \partial q^{2}$}

All the external lines should satisfy the on-shell condition when we use LoopTools. For this technical reason, LoopTools-2.12 cannot evaluate $C_{0 / 2}^{(Z / t)}$ and $D_{0 / 3}^{(t)}$ directly. In this case we need to convert this function to other functions. In this subsection, we express $C_{0 / 2}^{(Z / t)}$ and $D_{0 / 3}^{(t)}$ as combinations of $B_{0}$ and $\partial B_{0} / \partial q^{2}$.

$$
\begin{aligned}
C_{0}^{(Z)}= & \frac{\partial}{\partial m_{Z}^{2}} B_{0}\left(m_{A}^{2}, m_{Z}^{2}, m_{S}^{2}\right) \\
= & \frac{1}{m_{Z}^{4}+m_{S}^{4}+m_{A}^{4}-2 m_{Z}^{2} m_{S}^{2}-2 m_{A}^{2} m_{Z}^{2}-2 m_{A}^{2} m_{S}^{2}} \\
& \times\left[\left(m_{S}^{2}-m_{Z}^{2}+m_{A}^{2}\right)\left(-B_{0}\left(m_{A}^{2}, m_{Z}^{2}, m_{S}^{2}\right)+B_{0}\left(0, m_{Z}^{2}, m_{Z}^{2}\right)+2\right)-2 m_{S}^{2} \log \frac{m_{S}^{2}}{m_{Z}^{2}}\right] . \\
C_{2}^{(Z)}= & \frac{\partial}{\partial m_{Z}^{2}} B_{1}\left(m_{A}^{2}, m_{Z}^{2}, m_{S}^{2}\right)=\frac{\partial}{\partial m_{A}^{2}} B_{0}\left(m_{A}^{2}, m_{Z}^{2}, m_{S}^{2}\right) . \\
D_{0}^{(Z)}= & \frac{1}{2} \frac{\partial^{2}}{\partial\left(m_{Z}^{2}\right)^{2}} B_{0}\left(m_{A}^{2}, m_{Z}^{2}, m_{S}^{2}\right)
\end{aligned}
$$




$$
\begin{aligned}
= & \frac{2 m_{S}^{2} m_{A}^{2}}{\left(m_{Z}^{4}+m_{S}^{4}+m_{A}^{4}-2 m_{Z}^{2} m_{S}^{2}-2 m_{A}^{2} m_{Z}^{2}-2 m_{A}^{2} m_{S}^{2}\right)^{2}} \\
& \times\left[-B_{0}\left(m_{A}^{2}, m_{Z}^{2}, m_{S}^{2}\right)+B_{0}\left(0, m_{Z}^{2}, m_{Z}^{2}\right)+\frac{m_{Z}^{2}-m_{S}^{2}-m_{A}^{2}}{2 m_{A}^{2}} \log \frac{m_{S}^{2}}{m_{Z}^{2}}\right. \\
& \left.\quad-\frac{m_{A}^{6}-3\left(m_{Z}^{2}+m_{S}^{2}\right) m_{A}^{4}+3\left(m_{Z}^{2}-m_{S}^{2}\right)^{2} m_{A}^{2}-\left(m_{Z}^{2}+m_{S}^{2}\right)\left(m_{Z}^{2}-m_{S}^{2}\right)^{2}}{4 m_{Z}^{2} m_{S}^{2} m_{A}^{2}}\right] . \quad \text { E.28) } \\
D_{3}^{(Z)}= & \frac{1}{2} \frac{\partial^{2}}{\partial\left(m_{Z}^{2}\right)^{2}} B_{1}\left(m_{A}^{2}, m_{Z}^{2}, m_{S}^{2}\right) \\
= & \frac{1}{2} \frac{\partial}{\partial m_{A}^{2}} \frac{\partial}{\partial m_{Z}^{2}} B_{0}\left(m_{A}^{2}, m_{Z}^{2}, m_{S}^{2}\right) \\
= & \frac{m_{Z}^{2}+m_{S}^{2}-m_{A}^{2}}{\left(m_{Z}^{4}+m_{S}^{4}+m_{A}^{4}-2 m_{Z}^{2} m_{S}^{2}-2 m_{A}^{2} m_{Z}^{2}-2 m_{A}^{2} m_{S}^{2}\right)^{2}} \\
& \times\left(\left(m_{S}^{2}-m_{Z}^{2}+m_{A}^{2}\right)\left(-B_{0}\left(m_{A}^{2}, m_{Z}^{2}, m_{S}^{2}\right)+B_{0}\left(0, m_{Z}^{2}, m_{Z}^{2}\right)+2\right)-2 m_{S}^{2} \log \frac{m_{S}^{2}}{m_{Z}^{2}}\right) \\
& +\frac{1}{2} \frac{1}{m_{Z}^{4}+m_{S}^{4}+m_{A}^{4}-2 m_{Z}^{2} m_{S}^{2}-2 m_{A}^{2} m_{Z}^{2}-2 m_{A}^{2} m_{S}^{2}} \\
& \times\left(-B_{0}\left(m_{A}^{2}, m_{Z}^{2}, m_{S}^{2}\right)+B_{0}\left(0, m_{Z}^{2}, m_{Z}^{2}\right)+2-\left(m_{S}^{2}-m_{Z}^{2}+m_{A}^{2}\right) \frac{\partial}{\partial m_{A}^{2}} B_{0}\left(m_{A}^{2}, m_{Z}^{2}, m_{S}^{2}\right)\right) .
\end{aligned}
$$

Open Access. This article is distributed under the terms of the Creative Commons Attribution License (CC-BY 4.0), which permits any use, distribution and reproduction in any medium, provided the original author(s) and source are credited.

\section{References}

[1] ATLAS collaboration, Observation of a new particle in the search for the Standard Model Higgs boson with the ATLAS detector at the LHC, Phys. Lett. B 716 (2012) 1 [arXiv: 1207.7214] [INSPIRE].

[2] CMS collaboration, Observation of a new boson at a mass of $125 \mathrm{GeV}$ with the CMS experiment at the LHC, Phys. Lett. B 716 (2012) 30 [arXiv:1207.7235] [INSPIRE].

[3] G. Bertone, D. Hooper and J. Silk, Particle dark matter: Evidence, candidates and constraints, Phys. Rept. 405 (2005) 279 [hep-ph/0404175] [INSPIRE].

[4] N.G. Deshpande and E. Ma, Pattern of Symmetry Breaking with Two Higgs Doublets, Phys. Rev. D 18 (1978) 2574 [INSPIRE].

[5] R. Barbieri, L.J. Hall and V.S. Rychkov, Improved naturalness with a heavy Higgs: An Alternative road to LHC physics, Phys. Rev. D 74 (2006) 015007 [hep-ph/0603188] [INSPIRE].

[6] B.W. Lee and S. Weinberg, Cosmological Lower Bound on Heavy Neutrino Masses, Phys. Rev. Lett. 39 (1977) 165 [INSPIRE].

[7] M. Srednicki, R. Watkins and K.A. Olive, Calculations of Relic Densities in the Early Universe, Nucl. Phys. B 310 (1988) 693 [InSPIRE].

[8] P. Gondolo and G. Gelmini, Cosmic abundances of stable particles: Improved analysis, Nucl. Phys. B 360 (1991) 145 [InSPIRE]. 
[9] Z. Chacko, H.-S. Goh and R. Harnik, A twin Higgs model from left-right symmetry, JHEP 01 (2006) 108 [hep-ph/0512088] [INSPIRE].

[10] H.-S. Goh and C.A. Krenke, A Little Twin Higgs Model, Phys. Rev. D 76 (2007) 115018 [arXiv:0707.3650] [INSPIRE].

[11] E.M. Dolle and S. Su, Dark Matter in the Left Right Twin Higgs Model, Phys. Rev. D 77 (2008) 075013 [arXiv:0712.1234] [InSPIRE].

[12] J. Mrazek, A. Pomarol, R. Rattazzi, M. Redi, J. Serra and A. Wulzer, The Other Natural Two Higgs Doublet Model, Nucl. Phys. B 853 (2011) 1 [arXiv:1105.5403] [inSPIRE].

[13] E. Ma, Verifiable radiative seesaw mechanism of neutrino mass and dark matter, Phys. Rev. D 73 (2006) 077301 [hep-ph/0601225] [INSPIRE].

[14] M. Aoki, J. Kubo and H. Takano, Two-loop radiative seesaw mechanism with multicomponent dark matter explaining the possible $\gamma$ excess in the Higgs boson decay and at the Fermi LAT, Phys. Rev. D 87 (2013) 116001 [arXiv:1302.3936] [INSPIRE].

[15] Y. Kajiyama, H. Okada and T. Toma, Multicomponent dark matter particles in a two-loop neutrino model, Phys. Rev. D 88 (2013) 015029 [arXiv:1303.7356] [INSPIRE].

[16] E. Ma, Dark Scalar Doublets and Neutrino Tribimaximal Mixing from $A_{4}$ Symmetry, Phys. Lett. B 671 (2009) 366 [arXiv:0808.1729] [INSPIRE].

[17] E. Ma, Neutrino Mixing and Geometric CP-violation with $\Delta(27)$ Symmetry, Phys. Lett. B 723 (2013) 161 [arXiv:1304.1603] [INSPIRE].

[18] M. Hirsch, S. Morisi, E. Peinado and J.W.F. Valle, Discrete dark matter, Phys. Rev. D 82 (2010) 116003 [arXiv: 1007.0871] [inSPIRE].

[19] L. Lavoura, S. Morisi and J.W.F. Valle, Accidental Stability of Dark Matter, JHEP 02 (2013) 118 [arXiv: 1205.3442] [INSPIRE].

[20] T.A. Chowdhury, M. Nemevšek, G. Senjanović and Y. Zhang, Dark Matter as the Trigger of Strong Electroweak Phase Transition, JCAP 02 (2012) 029 [arXiv:1110.5334] [INSPIRE].

[21] D. Borah and J.M. Cline, Inert Doublet Dark Matter with Strong Electroweak Phase Transition, Phys. Rev. D 86 (2012) 055001 [arXiv:1204.4722] [INSPIRE].

[22] G. Gil, P. Chankowski and M. Krawczyk, Inert Dark Matter and Strong Electroweak Phase Transition, Phys. Lett. B 717 (2012) 396 [arXiv:1207.0084] [INSPIRE].

[23] J.M. Cline and K. Kainulainen, Improved Electroweak Phase Transition with Subdominant Inert Doublet Dark Matter, Phys. Rev. D 87 (2013) 071701 [arXiv:1302.2614] [INSPIRE].

[24] A. Ahriche and S. Nasri, Dark matter and strong electroweak phase transition in a radiative neutrino mass model, JCAP 07 (2013) 035 [arXiv: 1304.2055] [INSPIRE].

[25] T. Hambye and M.H.G. Tytgat, Electroweak symmetry breaking induced by dark matter, Phys. Lett. B 659 (2008) 651 [arXiv:0707.0633] [INSPIRE].

[26] J.-O. Gong, H.M. Lee and S.K. Kang, Inflation and dark matter in two Higgs doublet models, JHEP 04 (2012) 128 [arXiv: 1202.0288] [INSPIRE].

[27] Q.-H. Cao, E. Ma and G. Rajasekaran, Observing the Dark Scalar Doublet and its Impact on the Standard-Model Higgs Boson at Colliders, Phys. Rev. D 76 (2007) 095011 [arXiv:0708.2939] [INSPIRE]. 
[28] E. Dolle, X. Miao, S. Su and B. Thomas, Dilepton Signals in the Inert Doublet Model, Phys. Rev. D 81 (2010) 035003 [arXiv:0909.3094] [InSPIRE].

[29] X. Miao, S. Su and B. Thomas, Trilepton Signals in the Inert Doublet Model, Phys. Rev. D 82 (2010) 035009 [arXiv: 1005.0090] [inSPIRE].

[30] M. Gustafsson, S. Rydbeck, L. Lopez-Honorez and E. Lundstrom, Status of the Inert Doublet Model and the Role of multileptons at the LHC, Phys. Rev. D 86 (2012) 075019 [arXiv:1206.6316] [INSPIRE].

[31] M. Krawczyk, D. Sokolowska and B. Swiezewska, 2HDM with $Z_{2}$ symmetry in light of new LHC data, J. Phys. Conf. Ser. 447 (2013) 012050 [arXiv:1303.7102] [inSPIRE].

[32] M. Aoki, S. Kanemura and H. Yokoya, Reconstruction of Inert Doublet Scalars at the International Linear Collider, Phys. Lett. B 725 (2013) 302 [arXiv:1303.6191] [InSPIRE].

[33] A. Arhrib, R. Benbrik and T.-C. Yuan, Associated Production of Higgs at Linear Collider in the Inert Higgs Doublet Model, Eur. Phys. J. C 74 (2014) 2892 [arXiv:1401.6698] [INSPIRE].

[34] A. Arhrib, R. Benbrik and N. Gaur, $H \rightarrow \gamma \gamma$ in Inert Higgs Doublet Model, Phys. Rev. D 85 (2012) 095021 [arXiv:1201.2644] [InSPIRE].

[35] B. Swiezewska and M. Krawczyk, Diphoton rate in the inert doublet model with a $125 \mathrm{GeV}$ Higgs boson, Phys. Rev. D 88 (2013) 035019 [arXiv:1212.4100] [InSPIRE].

[36] M. Krawczyk, D. Sokolowska, P. Swaczyna and B. Swiezewska, Constraining Inert Dark Matter by $R_{\gamma \gamma}$ and WMAP data, JHEP 09 (2013) 055 [arXiv: 1305.6266] [INSPIRE].

[37] M. Gustafsson, E. Lundstrom, L. Bergstrom and J. Edsjo, Significant Gamma Lines from Inert Higgs Dark Matter, Phys. Rev. Lett. 99 (2007) 041301 [astro-ph/0703512] [InSPIRE].

[38] P. Agrawal, E.M. Dolle and C.A. Krenke, Signals of Inert Doublet Dark Matter in Neutrino Telescopes, Phys. Rev. D 79 (2009) 015015 [arXiv:0811.1798] [INSPIRE].

[39] S. Andreas, M.H.G. Tytgat and Q. Swillens, Neutrinos from Inert Doublet Dark Matter, JCAP 04 (2009) 004 [arXiv:0901.1750] [InSPIRE].

[40] C. Garcia-Cely and A. Ibarra, Novel Gamma-ray Spectral Features in the Inert Doublet Model, JCAP 09 (2013) 025 [arXiv: 1306.4681] [INSPIRE].

[41] D. Majumdar and A. Ghosal, Dark Matter candidate in a Heavy Higgs Model: Direct Detection Rates, Mod. Phys. Lett. A 23 (2008) 2011 [hep-ph/0607067] [InSPIRE].

[42] L. Lopez Honorez, E. Nezri, J.F. Oliver and M.H.G. Tytgat, The Inert Doublet Model: An Archetype for Dark Matter, JCAP 02 (2007) 028 [hep-ph/0612275] [INSPIRE].

[43] V. Silveira and A. Zee, Scalar phantoms, Phys. Lett. B 161 (1985) 136 [InSPIRE].

[44] J. McDonald, Gauge singlet scalars as cold dark matter, Phys. Rev. D 50 (1994) 3637 [hep-ph/0702143] [INSPIRE].

[45] C.P. Burgess, M. Pospelov and T. ter Veldhuis, The Minimal model of nonbaryonic dark matter: A Singlet scalar, Nucl. Phys. B 619 (2001) 709 [hep-ph/0011335] [INSPIRE].

[46] M. Klasen, C.E. Yaguna and J.D. Ruiz-Alvarez, Electroweak corrections to the direct detection cross section of inert Higgs dark matter, Phys. Rev. D 87 (2013) 075025 [arXiv: 1302.1657] [INSPIRE]. 
[47] A. Pierce and J. Thaler, Natural Dark Matter from an Unnatural Higgs Boson and New Colored Particles at the TeV Scale, JHEP 08 (2007) 026 [hep-ph/0703056] [INSPIRE].

[48] E. Lundstrom, M. Gustafsson and J. Edsjo, The Inert Doublet Model and LEP II Limits, Phys. Rev. D 79 (2009) 035013 [arXiv:0810.3924] [inSPIRE].

[49] E.M. Dolle and S. Su, The Inert Dark Matter, Phys. Rev. D 80 (2009) 055012 [arXiv: 0906.1609] [INSPIRE].

[50] D. Sokolowska, Dark Matter Data and Constraints on Quartic Couplings in IDM, arXiv:1107.1991 [INSPIRE].

[51] A. Goudelis, B. Herrmann and O. Stål, Dark matter in the Inert Doublet Model after the discovery of a Higgs-like boson at the LHC, JHEP 09 (2013) 106 [arXiv:1303.3010] [INSPIRE].

[52] A. Arhrib, Y.-L.S. Tsai, Q. Yuan and T.-C. Yuan, An Updated Analysis of Inert Higgs Doublet Model in light of the Recent Results from LUX, PLANCK, AMS-02 and LHC, JCAP 06 (2014) 030 [arXiv:1310.0358] [INSPIRE].

[53] T. Abe, R. Kitano and R. Sato, Discrimination of dark matter models in future experiments, arXiv: 1411.1335 [INSPIRE].

[54] L. Lopez Honorez and C.E. Yaguna, The inert doublet model of dark matter revisited, JHEP 09 (2010) 046 [arXiv: 1003.3125] [INSPIRE].

[55] Planck collaboration, P.A.R. Ade et al., Planck 2013 results. XVI. Cosmological parameters, Astron. Astrophys. 571 (2014) A16 [arXiv:1303.5076] [INSPIRE].

[56] L. Lopez Honorez and C.E. Yaguna, A new viable region of the inert doublet model, JCAP 01 (2011) 002 [arXiv: 1011.1411] [INSPIRE].

[57] J. Hisano, K. Ishiwata and N. Nagata, Gluon contribution to the dark matter direct detection, Phys. Rev. D 82 (2010) 115007 [arXiv:1007.2601] [INSPIRE].

[58] M.A. Shifman, A.I. Vainshtein and V.I. Zakharov, Remarks on Higgs Boson Interactions with Nucleons, Phys. Lett. B 78 (1978) 443 [INSPIRE].

[59] M.E. Peskin and D.V. Schroeder, An introduction to quantum field theory, Addison-Wesley, Reading, U.S.A. (1995).

[60] J. Pumplin, D.R. Stump, J. Huston, H.L. Lai, P.M. Nadolsky and W.K. Tung, New generation of parton distributions with uncertainties from global $Q C D$ analysis, JHEP 07 (2002) 012 [hep-ph/0201195] [INSPIRE].

[61] J. Hisano, K. Ishiwata and N. Nagata, A complete calculation for direct detection of Wino dark matter, Phys. Lett. B 690 (2010) 311 [arXiv:1004.4090] [INSPIRE].

[62] G. Bélanger, F. Boudjema, A. Pukhov and A. Semenov, MicrOMEGAs $s_{3}$ : A program for calculating dark matter observables, Comput. Phys. Commun. 185 (2014) 960 [arXiv: 1305.0237] [INSPIRE].

[63] LUX collaboration, D.S. Akerib et al., First results from the LUX dark matter experiment at the Sanford Underground Research Facility, Phys. Rev. Lett. 112 (2014) 091303 [arXiv: 1310.8214] [INSPIRE].

[64] XENON1T collaboration, E. Aprile, The XENON1T Dark Matter Search Experiment, Springer Proc. Phys. C12-02-22 (2013) 93 [arXiv:1206.6288] [INSPIRE]. 
[65] J.L. Feng et al., Planning the Future of U.S. Particle Physics (Snowmass 2013): Chapter 4: Cosmic Frontier, arXiv:1401.6085 [INSPIRE].

[66] J. Billard, L. Strigari and E. Figueroa-Feliciano, Implication of neutrino backgrounds on the reach of next generation dark matter direct detection experiments, Phys. Rev. D 89 (2014) 023524 [arXiv: 1307.5458] [InSPIRE].

[67] J. Hisano, K. Ishiwata, N. Nagata and T. Takesako, Direct Detection of Electroweak-Interacting Dark Matter, JHEP 07 (2011) 005 [arXiv:1104.0228] [INSPIRE].

[68] V.A. Novikov, M.A. Shifman, A.I. Vainshtein and V.I. Zakharov, Calculations in External Fields in Quantum Chromodynamics. Technical Review, Fortsch. Phys. 32 (1984) 585 [INSPIRE].

[69] G. Passarino and M.J.G. Veltman, One Loop Corrections for $e^{+} e^{-}$Annihilation Into $\mu^{+} \mu^{-}$ in the Weinberg Model, Nucl. Phys. B 160 (1979) 151 [INSPIRE].

[70] T. Hahn and M. Pérez-Victoria, Automatized one loop calculations in four-dimensions and D-dimensions, Comput. Phys. Commun. 118 (1999) 153 [hep-ph/9807565] [InSPIRE]. 